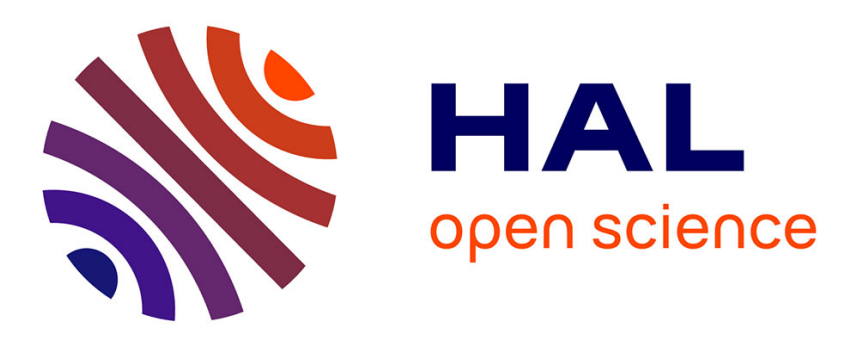

\title{
Model-based analysis of myocardial strain data acquired by tissue Doppler imaging.
}

Virginie Le Rolle, Alfredo Hernandez, Pierre-Yves Richard, Erwan Donal, Guy Carrault

\section{- To cite this version:}

Virginie Le Rolle, Alfredo Hernandez, Pierre-Yves Richard, Erwan Donal, Guy Carrault. Modelbased analysis of myocardial strain data acquired by tissue Doppler imaging.. Artificial Intelligence in Medicine, 2008, 44 (3), pp.201-219. 10.1016/j.artmed.2008.06.001 . inserm-00336116

\section{HAL Id: inserm-00336116 https://www.hal.inserm.fr/inserm-00336116}

Submitted on 17 Sep 2009

HAL is a multi-disciplinary open access archive for the deposit and dissemination of scientific research documents, whether they are published or not. The documents may come from teaching and research institutions in France or abroad, or from public or private research centers.
L'archive ouverte pluridisciplinaire HAL, est destinée au dépôt et à la diffusion de documents scientifiques de niveau recherche, publiés ou non, émanant des établissements d'enseignement et de recherche français ou étrangers, des laboratoires publics ou privés. 
3 Model-Based Analysis of Myocardial Strain Data acquired by Tissue Doppler Imaging

6 Virginie Le Rolle ${ }^{12}$, Alfredo I. Hernández ${ }^{12}$, Pierre-Yves Richard $^{4}$, Erwan Donal ${ }^{123}$ and Guy Carrault ${ }^{12}$ 7

$8 \quad{ }^{1}$ INSERM U642, Rennes, F-35000, France

$9 \quad{ }^{2}$ Université de Rennes 1, LTSI, Rennes, F-35000, France.

$10 \quad{ }^{3}$ CHU Rennes, Department of Cardiology, Rennes, F-35000, France

$11{ }^{4}$ Supelec-IETR, Rennes, France.

12

13 email:

14 virginie.lerolle@univ-rennes1.fr

15 tel:

$16 \quad$ (33) 223235585

17 fax:

$18 \quad$ (33) 223236917

19 address :

20 LTSI, Campus de Beaulieu,

21 Université de Rennes 1,

22263 Avenue du Général Leclerc

23 CS $74205-35042$ Rennes Cedex, France. 
25 Summary:

26 Objective: Tissue Doppler Imaging (TDI) is commonly used to evaluate regional ventricular contraction 27 properties through the analysis of myocardial strain. During the clinical examination, a set of strain signals is 28 acquired concurrently at different locations. However, the joint interpretation of these signals remains 29 difficult. This paper proposes a model-based approach in order to assist the clinician in making an analysis of 30 myocardial strain.

31 Methods and material: The proposed method couples a model of the left ventricle, which takes into account 32 cardiac electrical, mechanical and hydraulic activities with an adapted identification algorithm, in order to 33 obtain patient-specific model representations. The proposed model presents a tissue-level resolution, adapted 34 to TDI strain analysis. The method is applied in order to reproduce TDI strain signals acquired from two 35 healthy subjects and a patient presenting with Dilated Cardiomyopathy (DCM).

36 Results: The comparison between simulated and experimental strains for the three subjects reflects a 37 satisfying adaptation of the model on different strain morphologies. The mean error between real and 38 synthesized signals is equal to $2.34 \%$ and $2.09 \%$, for the two healthy subjects and $1.30 \%$ for the patient 39 suffering from DCM. Identified parameters show significant electrical conduction and mechanical activation 40 delays for the pathologic case and have shown to be useful for the localization of the failing myocardial 41 segments, which are situated on the anterior and lateral walls of the ventricular base.

42 Conclusion: The present study shows the feasibility of a model-based method for the analysis of TDI strain 43 signals. The identification of delayed segments in the pathologic case produces encouraging results and may 44 represent a way to better utilize the information included in strain signals and to improve the therapy 45 assistance.

46 Index Terms - Biomedical Systems modeling, Biomedical Model Simulation, Model-Based 47 Interpretation, Echocardiography 


\section{Introduction}

50 In the daily clinical practice, Doppler Echocardiography has become a fundamental method in evaluating the cardiac function. This non-invasive technique is particularly useful for the analysis of blood flow and cardiac anatomy, facilitating the diagnosis of cardiovascular pathologies. Tissue Doppler Imaging (TDI) is a more recent tool that can be useful in assessing regional myocardial deformations through the estimation of regional myocardial strain [1,2] and strain rate [3]. Myocardial strain analysis has shown to be useful, for example, in differentiating healthy and ischemic myocardium $[4,5]$. More details on TDI acquisition and analysis are presented in appendix A.

A typical experimental strain signal is shown in figure 1. Tissue extensions correspond with positive strains while tissue compressions result in negative strains. This signal reflects the different phases occurring during the cardiac cycle (figure 1): $i$ ) the isovolumic contraction phase; ii) the systole; iii) the isovolumic relaxation; iv) the diastole, which can be divided into three periods: the first one corresponding to a rapid filling phase due to an aspiration of blood inside the ventricle, a slower filling period and the late diastolic filling phase, corresponding to the atrial systole. Although TDI presents some disadvantages such as a dependence on the ultrasound beam orientation [1], that affects the reproducibility, and the presence of noise [2], it has been successfully validated by the comparison with other imaging methods, like MRI or sonomicrometry [2, 6]. The main advantages of TDI are related to the possibility of obtaining real-time measurements and the relative low cost of this technique.

\section{1)}

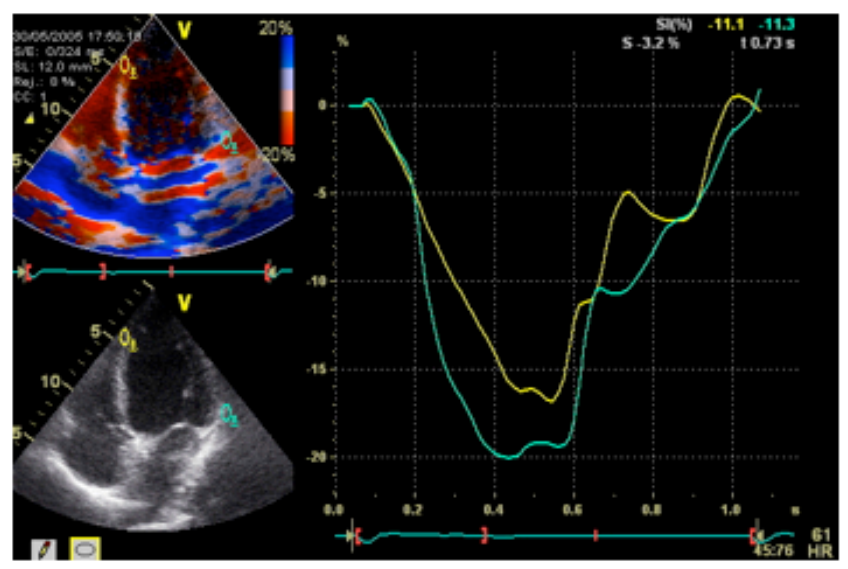

67 and atrial systole (A)

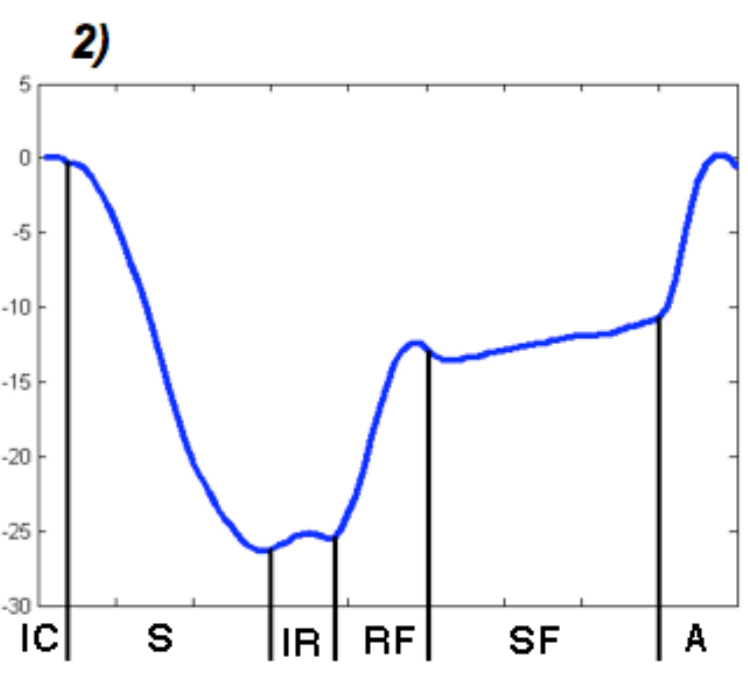

Figure 1: 1) Tissue Doppler Imaging applied to a four-cavity echocardiographic acquisition. 2) A typical experimental strain of a segment can be divided in several phases: isovolumic contraction (IC), ejection (S), isovolumic relaxation (IR), rapid filling (RF), slow filling period (SF)

Several indicators can be derived from strain estimations, such as maximum systolic velocity or ventricular filling period $[4,7]$. Although the use of such indicators has already shown interesting results in identifying some cardiovascular pathologies, the whole information contained in the strain signal is not yet fully utilized. 
The analysis of the morphology of a set of strain signals, acquired concurrently at different regions of the myocardium, is a very difficult task. This is partly due to the multidimensionality of the problem and the fact that the different processes which lead to ventricular contraction (mechano-hydraulic interactions, electrical activation and propagation, etc.) have to be jointly considered for an appropriate interpretation.

In this paper we propose a model-based approach to assist the clinician on the analysis of myocardial strain signals. A brief presentation of current approaches for modeling the electromechanical activity of the heart is proposed in section 2. Section 3 describes the proposed ventricular model and the parameter identification method. Finally, in section 4, the results of patient-specific identifications and the analysis of strain morphologies are presented and discussed.

\section{Current electromechanical models of the cardiac function}

A variety of mathematical models of the ventricular function have been proposed in other studies. The simplest models are based on a time-varying elastance $[8,9]$. These overall, lumped-parameter models give realistic simulations of cardiac pressure and volume and require low computational resources. However, as the whole left ventricle (LV) is represented as a single element, it is not possible to analyze the regional ventricular function. Other approaches have been proposed in order to represent explicitly, at many different levels of detail, the cardiac electrical activity, the excitation-contraction coupling, the mechanical activity and the mechano-hydraulic coupling. The main modeling techniques applied to represent these activities are briefly recalled in the following paragraph.

\subsection{Electrical Activity}

Models of the cardiac electrical activity, defined at the cell level, can be classified into three categories:

- Biophysical continuous models, composed of detailed descriptions of the cardiac action-potential, based on the Hudking-Huxley formulation [10], and presenting different levels of detail on the ionic currents included [11-13].

- Phenomenological continuous models, which reproduce qualitatively the electrical activation without describing the different ion channels. They present less computational requirements and result in simplified representations of the model's electrical activity (often based on the FitzHughNagumo model [14]).

- Simplified discrete models, they are often based on cellular automata, representing the different electrical states of a myocyte's action potential [15].

Coupling of cell-level continuous models in order to represent a patch of myocardial tissue, or more complex geometries such as the whole LV, can be performed by using the monodomain or bidomain approaches [16, 17]. Cellular automata are typically coupled by means of a discrete flag transmitted to all neighbors during the depolarization of each element [18]. Another approach to represent the propagation of the cardiac electrical activity is limited to modeling the evolution of the depolarization wavefront through the cardiac tissue, described by the eikonal diffusion equation [19]. 


\subsection{Mechanical Activity}

10 The ventricular mechanical activity is usually described as a function of its active and passive properties. 11 Active properties are the consequence of the shortening and lengthening of sarcomeres, which are the 12 elementary mechanical contractile elements of myocytes. This mechanical activity is under the influence of 13 an electrical activity, since the variation of calcium concentration during the action potential allows the 14 development of force. Passive properties are mainly related to fiber structure and orientation, collagen 15 properties and metabolic conditions (such as hypoxia or ischemia).

Models of the active properties include:

- Huxley-type models, which represent changes of conformation on sarcomeres as a function of crossbridge position [20] and intracellular calcium concentration [21].

- Hill's models, which use one of the forms of the original Hill's force-length relation [22-24] or the modified Hill equation, which is a sigmoid function relating calcium concentration and active tension.

Passive myocardial properties are mainly described through specific mechanical constitutive laws. Most of these mechanical laws are hyperelastic, incompressible and anisotropic [28-30]. The majority of them have been determined using uniaxial [31] or biaxial tension tests [30]. An empiric law based on the description of sarcomere dynamics has been proposed by [22].

The simulation of these models are often based on finite-element methods (FEM) [32-35]. Although this kind of formulation allows a rather detailed description of the myocardium dynamics, it requires significant computational resources. Another complementary approach for modeling the myocardium deformation is based on a mass-spring system [36] activated by a model of the electrical activity through a simplified electro-mechanical coupling.

\subsection{Fluid-structure interaction and boundary conditions}

The simulation of a realistic cardiac cycle requires the representation of the interaction between the myocardium and the blood inside the heart's chambers and in the systemic and pulmonary circulations. Several approaches to this have been proposed:

- Fluid-structure interaction models integrate simultaneous descriptions of the fluid inside the cavity, by means of a Navier-Stokes formulation $[37,38]$ and the myocardial wall motion, based on the 
description of a constitutive law, as presented in the previous section.

- Rule-based definition of boundary conditions: In most FEM models of the LV, boundary conditions are imposed by the intra-ventricular pressure, by means of a set of rules defining different levels of pressure for each phase of the cardiac cycle. The ejection and filling phase can be deduced from a simplified model of the circulation [32, 34], while a penalty pressure is applied during the isovolumetric phases, in order to keep the volume constant [39]. An alternative method has been proposed in a recent paper, combining a lumped parameter model of the circulation with an FEM model of the ventricles, based on the estimation of an equivalent overall ventricular elastance for each time-step [40]. These approaches imply the uniformity of blood pressure inside the cavity. However, Courtois et al have shown the importance of regional pressure gradients in the LV, especially during diastole [41].

\subsection{Model-based analysis of strain signals}

Complete models of ventricular activity are developed from a combination of the several different approaches previously described and some of these models have been applied to the analysis of myocardial strain. Nickerson et al [42] proposed a 3D model of cardiac electromechanics and used the fiber extension ratio to study the role of electrical heterogeneity in the cardiac function. The model includes a precise description of ventricular contraction, but requires around three weeks of simulation time on a parallel computer in order to reproduce one beat. Kerckhoffs et al [27] have proposed a 3D finite element model of cardiac mechanics used to compare synthesized ventricular strain signals generated by a normal and a synchronous electrical activation sequence. The model simulations show unphysiological contraction patterns when a physiological electrical activation is applied. Results illustrate the difficulty of using such a model in a concrete clinical application and the necessity of representing heterogeneous electromechanical couplings. The same model has been used by Ubbink et al [43] to study circumferential and circumferentialradial shear strain. Simulated strains are compared to Magnetic Resonance Tagging (MRT) data. The modelbased approach helps in determining the influence of fiber angle on regional contraction. However, as the model includes simplified descriptions of its hydraulic activity, the whole morphology of ventricular strain cannot be analyzed, especially during the rapid filling phases.

As previously stated, the main objective of this study is to propose a personalized model-based approach in order to assist the clinicians in the interpretation of myocardial strain signals measured with Tissue Doppler echocardiography. The models described in the previous paragraphs are difficult to apply in this case, as they are characterized by a significant number of parameters, require significant computational resources and, consequentially, their ability to be identified is more complex. Previous studies [44] have shown the benefit of combining a minimal cardiovascular model and an identification algorithm for real-time patient specific modeling and diagnosis in the case of a pulmonary embolism. As the high resolution of an FEM model is not 
necessary for this particular study, a new model has thus been developed, with the following specific properties:

- The model resolution has been adapted to the problem, keeping a similar abstraction level as the experts for the analysis of strain signals.

- The model is based on a functional integration of interacting physiological processes, by taking into account: i) the electro-mechanical coupling, ii) the interactions between the myocardial wall and the blood inside the cavity and iii) a simplified representation of the systemic circulation. This allows the representation of the main cardiac properties required to tackle the problem under study, like the Frank-Starling law and the influence of preload and afterload.

The next section presents a detailed description of the proposed model and the identification algorithm which is applied in order to obtain patient-specific model parameters.

\section{Materials and methods}

\subsection{Model description}

\subsubsection{General presentation of the proposed model}

In order to simulate the strain measured for each myocardial region, the proposed LV model has been divided into twelve segments, composed of three layers at the basal, equatorial and apical level [45]. Each layer is separated into 4 components: septal, lateral, anterior and inferior wall (figure 2). Each wall segment interacts with the blood inside the corresponding intra-ventricular cavity (which is itself segmented in a consistent manner). It should be noticed that such a 12-wall segmentation does not correspond to the standard myocardial segmentation defined in [46]. However, this segmentation has already been used in different studies (such as in [45]) and remains significant from a clinical standpoint.

The LV has been approximated by a truncated ellipsoid as it has already produced encouraging results in other studies, for the analysis of the electrical propagation during contraction [19, 47] or ventricular torsion [48]. The choice concerning the modeling formalism for each cardiac activity are summarized here:

- Electrical activity: A cellular automata network has been chosen to describe the electrical activation sequence for the 12 segments. This formalism presents low computational costs, while describing the basic properties of the cardiac electrical activity.

- Mechanical activity: The myocardium has been supposed to be hyperelastic, incompressible and transverse isotropic. The twist motion of the ventricle has been neglected, as it cannot be measured with tissue Doppler echocardiography. As circumferential and longitudinal strains are less sensitive to cardiac fiber angle [43], only a mean fiber angle is taken into account.

- Hydraulic activity: A lumped parameter model is used to describe the hydraulic activity of the intraventricular cavity and the influence of preload and afterload. This representation is adapted to 
reproduce major cardiac properties like the Frank-Starling law and the representation of regional pressure gradient that has been observed in the LV [41].

:17 The proposed model can be seen as an improvement of elastance models, by representing a set of sub-pumps interconnected in the hydraulic domain and commanded by a coordinated electrical activity. Each pump represents a macroscopic, tissue-level segment of the LV wall.

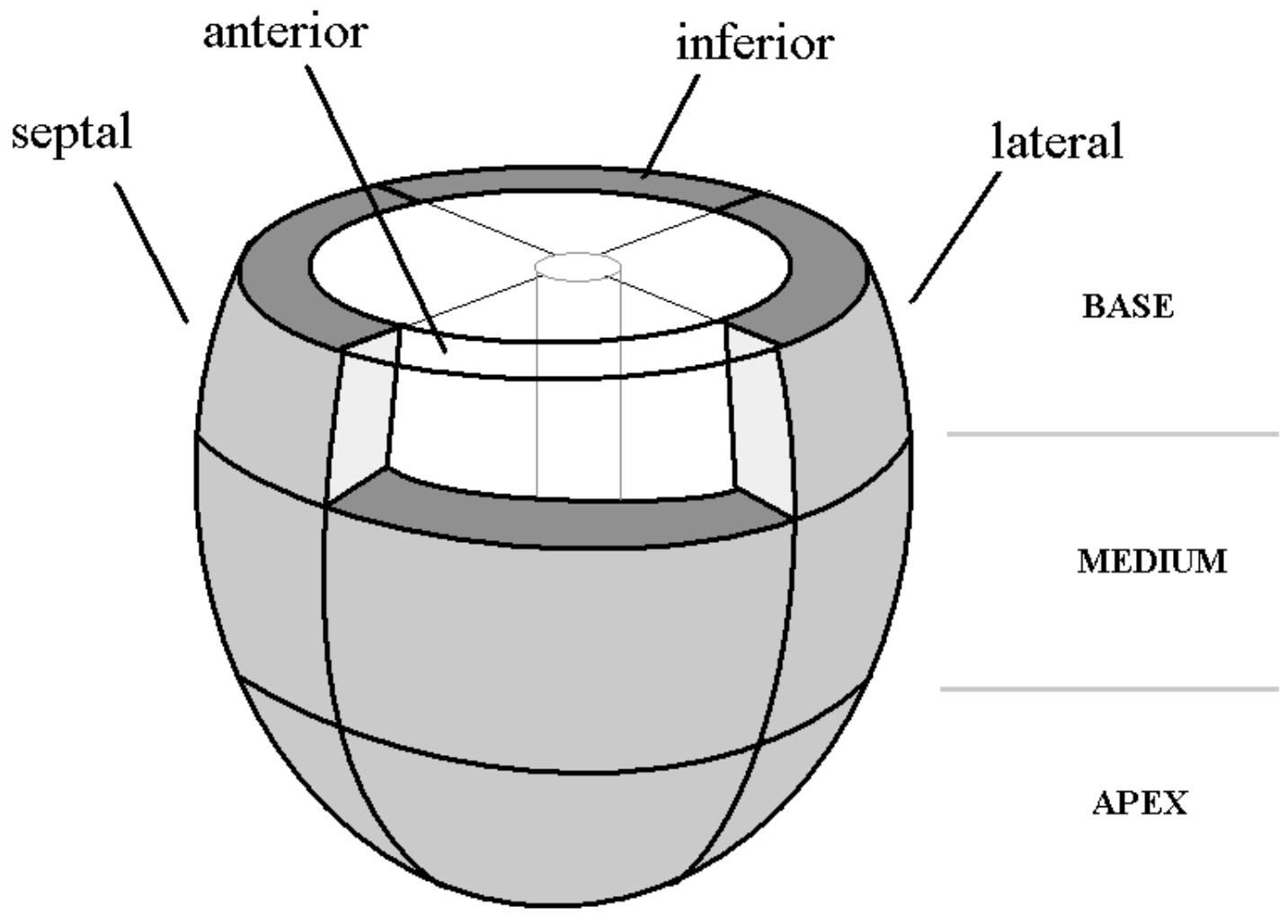

Figure 2: Ventricular model segmentation. The model is composed of 12 segments, corresponding to the septal, lateral, anterior and inferior

\subsubsection{Electrical activation model}

The ventricle has been represented by twelve cellular automata to describe the electrical propagation during contraction. Each automaton is defined by four electrical states [49, 50] (figure 3): $i$ ) rapid depolarization period $(R D P)$, ii) absolute refractory period $(A R P)$, iii) relative refractory period $(R R P)$ and $i v)$ a waiting period (idle). The transitions between states happen spontaneously at the end of the duration of each phase or due to an external activation, during the idle or $R R P$ states. After the $R D P$ period, each automaton transmits a stimulus to its neighboring segments. Each automaton is fully connected (antegrade and retrograde connections) to its three or four neighbors. An external excitation first stimulates the mid-septum segment and is then propagated to the other segments in function of each automaton's parameter values. 


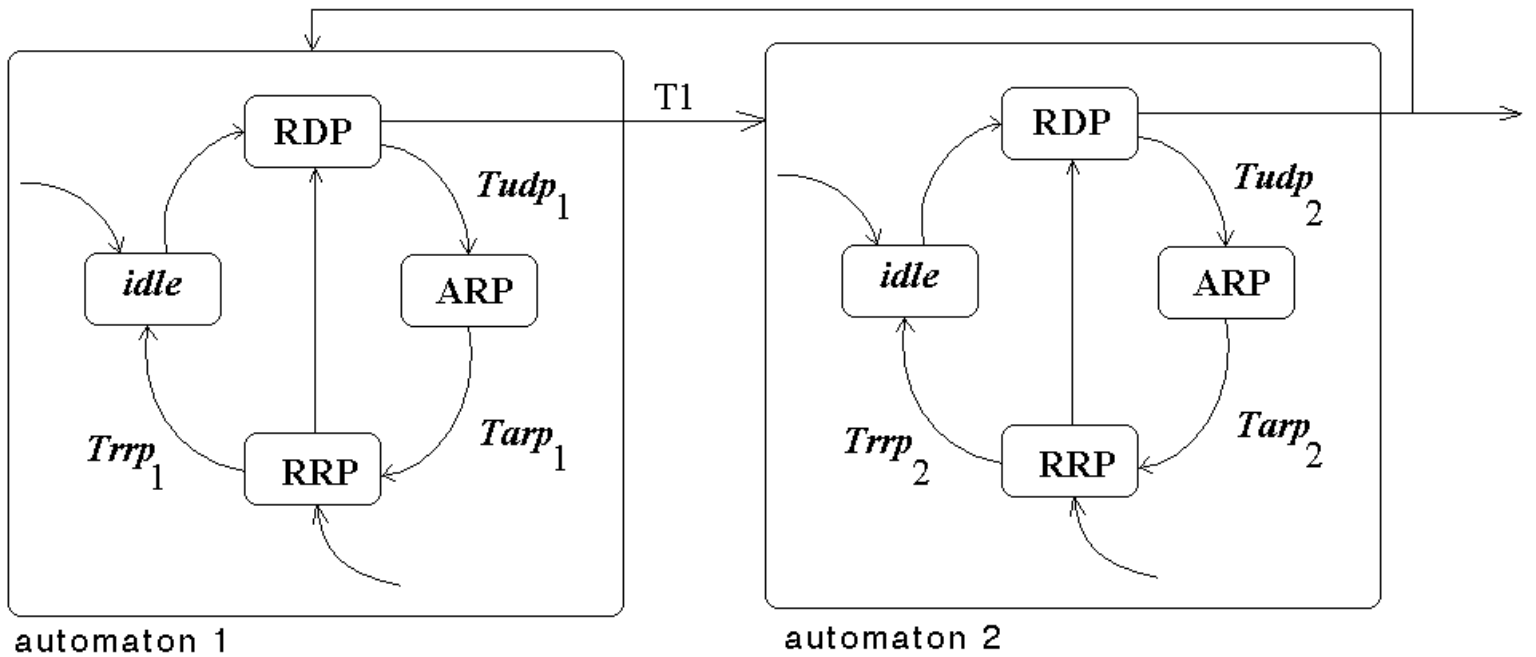

automaton 1

automaton 2

Figure 3: States and coupling conditions for cellular automata models. Each automaton is characterized by four electrical states: $i)$ rapid depolarization period $(R D P)$, ii) absolute refractory period $(A R P)$, iii) relative refractory period $(R R P)$ and $i v)$ a waiting period (idle).

Electrical activation has been described as an anisotropic propagation [51], as the conduction delay is about three times longer in the horizontal direction than in the vertical direction. The coupling between two cellular automata is defined by the period $T_{1}$ for the antegrade link and the period $T_{2}$ for the retrograde link (see figure 3). For a vertical coupling, these periods are defined as $\mathrm{T}_{1}=\operatorname{Tr} d p_{1}$ and $\mathrm{T}_{2}=\operatorname{Tr} d p_{2}$. For a horizontal coupling, they are equal to $\mathrm{T}_{1}=3 \cdot \operatorname{Tr} d p_{1}$ and $\mathrm{T}_{2}=3 \cdot \operatorname{Tr} d p_{2}$.

\subsubsection{Mechanical-hydraulic model}

The radial force developed by each segment is computed by integrating the radial stress on the wall surface. Myocardial stress is usually expressed as the sum of active and passive stresses.

$$
F_{r}=\int \sigma_{r} \cdot d S=\int\left(\sigma_{r a}+\sigma_{r p}\right) \cdot d S
$$

The active stress can be expressed using the relation:

$$
\sigma_{a}=T_{a} \cdot(F N) .(F N)^{T}
$$

where $F$ is the deformation gradient tensor, $N$ stands for a unitary vector in the fiber direction and $T_{a}$ is the active tension. As in other studies [26, 27], $T_{a}$ is approached by means of a trigonometric function. In this case, the $T_{a}$ function is inspired from the studies of Hunter et al [25] and is defined as:

$$
T_{a}=T_{r e f} \frac{\left[\mathrm{Ca}^{2+}\right]_{i}^{n}}{\left[\mathrm{Ca}^{2+}\right]_{i}^{n}+C a_{50}^{n}}[1+\beta(\lambda-1)]
$$

where $T_{r e f}$ is the value of the tension at $\lambda=1, C a_{50}$ the calcium concentration at $50 \%$ of the isometric tension, $n$ is the Hill coefficient determining the shape of the curve and $\beta$ is the myofilament "cooperativity". The parameters values and functions for $\mathrm{Ca}_{50}$ and $n$ are taken from [25]. [ $\left.\mathrm{Ca}^{2+}\right]_{i}$ is the intracellular calcium concentration, which represents the mechanical activation level, and is defined in this model as: 


$$
\left[\mathrm{Ca}^{2+}\right]_{i}=\left\{\begin{array}{cc}
0 & t_{e s}<0 \\
K \sin \left(\pi \cdot t_{e s} / T_{\max }\right) & 0 \leq t_{e s} \leq T_{\max } \\
0 & t_{e s}>T_{\max }
\end{array}\right.
$$

Where tes is the time elapsed since the end of the $R D P$ for each segment $s, T_{\max }$ the activation duration and $K$ the maximum level of calcium concentration. The deformation gradient tensor $F$ can be defined by characterizing the myocardial motion in spherical coordinates. Supposing that a material particle in the undeformed state $(R, \Theta, \Psi)$ goes to $(r, \theta, \varphi)$ in the deformed state, a radial deformation can be described by:

$$
r=r(R) ; \quad \theta=\Theta ; \quad \varphi=\Psi
$$

These relations define a square diagonal matrix $F$ that includes the strains $\lambda_{r}, \lambda_{\theta}$ and $\lambda_{\varphi}$ in the three principal directions. It can easily be shown that the deformations in these directions are expressed as: $\lambda_{r}=\partial r / \partial R$ and $\lambda_{\theta}=\lambda_{\varphi}=r / R$, the latter common value being usually denoted by $\lambda$. Additionally, the myocardium is supposed to be incompressible, which is a classical assumption for the cardiac muscle, and the following additional property holds: $\operatorname{det}(F)=1$. So a simple relation between $\lambda_{r}$ and $\lambda$ can be found: $\lambda_{r}=1 / \lambda^{2}$.

The passive stress is due to myocardium organization (fibers, collagen...) and can be expressed using the equation:

$$
\sigma_{p}=-p I+2 F \frac{\partial W}{\partial C} F^{T}
$$

where $W$ is the strain energy function, $p$ stands for the hydrostatic pressure, $I$ is the identity matrix and $C$ is the Cauchy-Green tensor computed from $F$ through $C=F^{T} F$. The energy function used in this paper is the one defined by Humphrey et al. [28], which is a polynomial energy function. The polynomial form facilitates the implementation and has shown its efficiency in many studies [48, 52]:

$$
W=c_{1}(\alpha-1)^{2}-c_{2}(\alpha-1)^{3}+c_{3}\left(I_{1}-3\right)+c_{4}\left(I_{1}-3\right)(\alpha-1)+c_{5}\left(I_{1}-3\right)^{2}
$$

Where $I_{1}$ and $\alpha=\sqrt{I_{4}}$ stand for the invariants of the deformation gradient tensor. Finally, the passive stress can be expressed as:

$$
\sigma_{p}=-p I+2 W_{1} B+2 W_{4} F N \times N F^{t}
$$

with $W_{1}=\partial W / \partial I_{1}, W_{4}=\partial W / \partial I_{4}, I_{1}=\operatorname{tr}(C)$ and $I_{4}=N^{t} . C . N$

Since the total stress tensor has been defined as the sum of the active and passive ones, the three directions components are:

$$
\begin{gathered}
\sigma_{r}=-p+\tilde{\sigma}_{r} \\
\sigma_{\theta}=-p+\tilde{\sigma}_{\theta} \\
\sigma_{\varphi}=-p+\tilde{\sigma}_{\varphi}
\end{gathered}
$$

$$
\text { where } \tilde{\sigma}_{r}=2 \cdot W_{1} / \lambda^{4},
$$$$
\tilde{\sigma}_{\theta}=2 \cdot W_{1} \cdot \lambda^{2}+2 \cdot W_{4} \cdot \lambda^{2} \cdot \cos (\psi)+T_{a} \cdot W_{4} \cdot \lambda^{2} \cdot \cos (\psi)
$$$$
\tilde{\sigma}_{\varphi}=2 \cdot W_{1} \cdot \lambda^{2}+2 \cdot W_{4} \cdot \lambda^{2} \cdot \sin (\psi)+T_{a} \cdot W_{4} \cdot \lambda^{2} \cdot \sin (\psi)
$$ 
This equation system is implicit and the Laplace Relation is added to link the three stress components. This relation has been demonstrated for a thin ellipsoidal myocardial segment in [53], which shows that the thin wall theory is adequate for the estimation of average longitudinal and latitudinal stresses in ventricular walls:

$$
-\sigma_{r}=\frac{\sigma_{\theta} \times e}{R_{\theta}}+\frac{\sigma_{\varphi} \times e}{R_{\varphi}}
$$

:91 where $e$ is the wall thickness, $R_{\varphi}$ and $R_{\theta}$ stand for the radii of curvature in the meridian and parallel directions. As the ventricle is assumed to be an ellipsoid of revolution, $\left(R_{\varphi}, R_{\theta}\right)$ can be expressed as:

$$
\begin{aligned}
& R_{\varphi}=\frac{\left(a^{2} \cdot \cos ^{2} \varphi+b^{2} \cdot \sin ^{2} \varphi\right)^{3 / 2}}{a b} \\
& R_{\theta}=\frac{a}{b}\left(a^{2} \cdot \cos ^{2} \varphi+b^{2} \cdot \sin ^{2} \varphi\right)^{1 / 2}
\end{aligned}
$$

Since $\sigma_{r}$ can be computed, the wall radial force can be obtained by integrating the radial stress on the segment surface:

$$
F_{r}=-\int \sigma_{r} d S=\tilde{\sigma}_{\theta} \cdot \lambda^{2} \cdot K_{\theta}+\tilde{\sigma}_{\varphi} \cdot \lambda^{2} \cdot K_{\varphi}-\tilde{\sigma}_{r} \cdot \lambda^{2} \cdot K_{r}
$$

998 with

$$
K_{\theta}=\iint \frac{R^{2} \sin \varphi}{1+\frac{R_{\varphi}}{e}+\frac{R_{\varphi}}{R_{\theta}}} \cdot d \varphi \cdot d \theta, \quad K_{\varphi}=\iint \frac{R^{2} \sin \varphi}{1+\frac{R_{\varphi}}{e}+\frac{R_{\varphi}}{R_{\theta}}} d \varphi \cdot d \theta, \quad K_{r}=\iint \frac{e \cdot R_{\varphi}+e \cdot R_{\theta}}{e \cdot R_{\varphi}+e \cdot R_{\theta}+R_{\varphi} \cdot R_{\varphi}} \cdot R^{2} \cdot \sin \varphi \cdot d \varphi \cdot d \theta
$$

This last relation provides the constitutive law suitable to model the segment.

\subsubsection{Mechano-Hydraulic coupling}

The mechano-hydraulic interaction between the myocardial wall and the blood inside the ventricular cavity is characterized by the coupling relation:

$$
P_{S}=\frac{F_{r}}{S}
$$

where $P_{S}$ is the pressure on the wall surface, $F_{r}$ is the radial force developed by the wall segment and $S$ can be easily calculated since the surface is ellipsoidal.

\subsubsection{Hydraulic description}

The blood behavior inside the cardiac cavity should also be described. Indeed, although the flow $Q$ is supposed to remain the same in each cavity segment, the pressure varies from the wall surface $\left(P_{S}\right)$ to the i11 cavity center $\left(P_{c}\right)$. This variation is partially due to blood viscosity. So a hydraulic resistance $R$ is defined. :12 To describe the rapid filling phases, this resistance is considered lower during the diastole $\left(R_{\min }\right)$ and higher $: 13$ during systole $\left(R_{\max }\right)$. A resistive law relates pressure and flow:

$$
Q=\frac{P_{c}-P_{s}}{R}
$$

:15 It is also necessary to take into account the blood mass effects which bring some inertial properties. So a hydraulic inertance $I$ can be introduced in order to define the law: 


$$
P_{c}-P_{s}=I \frac{d Q}{d t}
$$

:18 To summarize, each ventricular segment is modeled by four distinct entities for the electrical, mechanical, i19 mechano-hydraulic coupling and hydraulic parts. Each part is composed of the equations previously :20 described and some input-output relations. The links between the different parts of the segment model are i21 graphically described in figure 4.
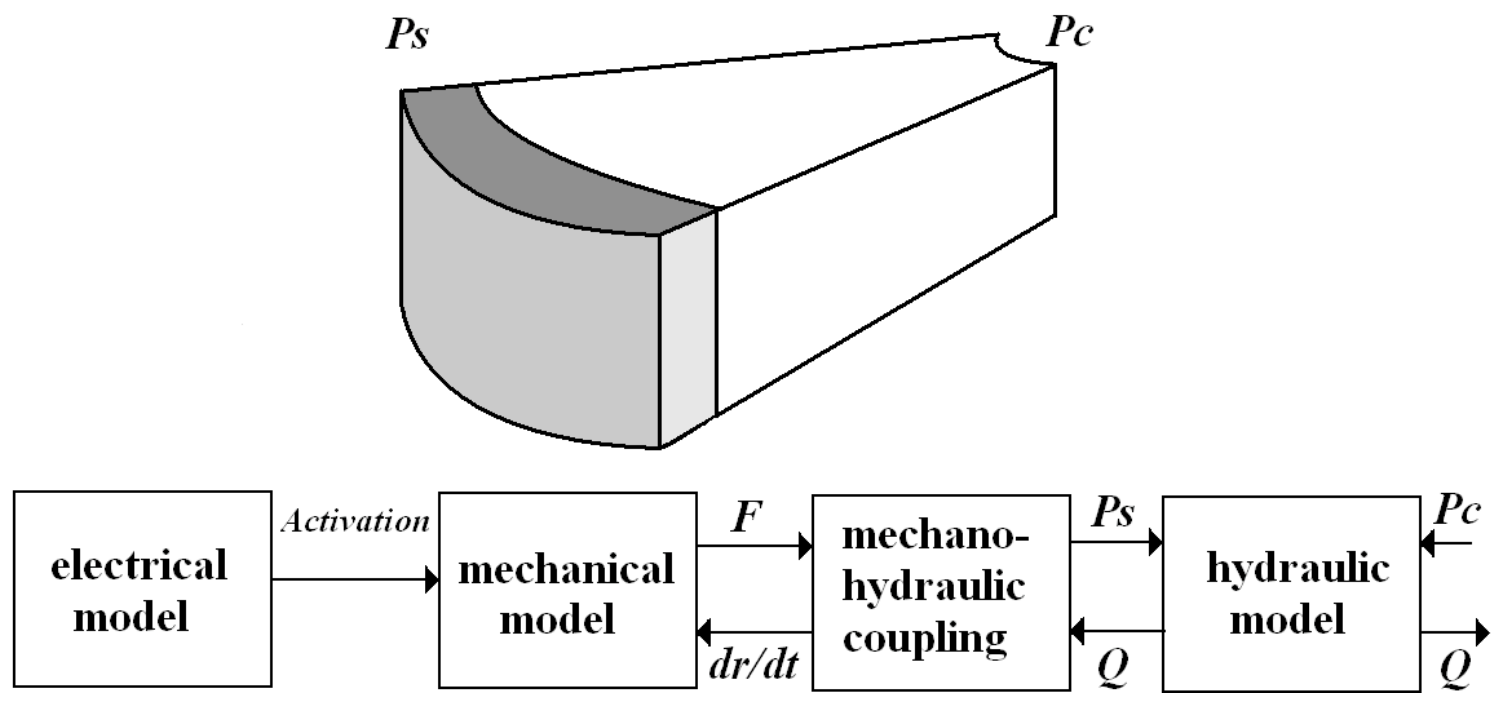

Figure 4: Model of each ventricular segment. The mechanical model is connected to the electrical model and the mechano-hydraulic coupling entity respectively through a simplified calcium concentration $([\mathrm{Ca} 2+])$ representation, the radial force $(F)$ and velocity $(d r / d t)$. The hydraulic model is linked to the mechano-hydraulic coupling entity and to the other segments respectively through the wall surface pressure ( $P s)$, the flow $(Q)$, and the pressure at the center of the cavity $\left(P_{c}\right)$.

i31 The connections between the three layers are defined by considering the resistive properties of the blood i32 inside the cavity. 

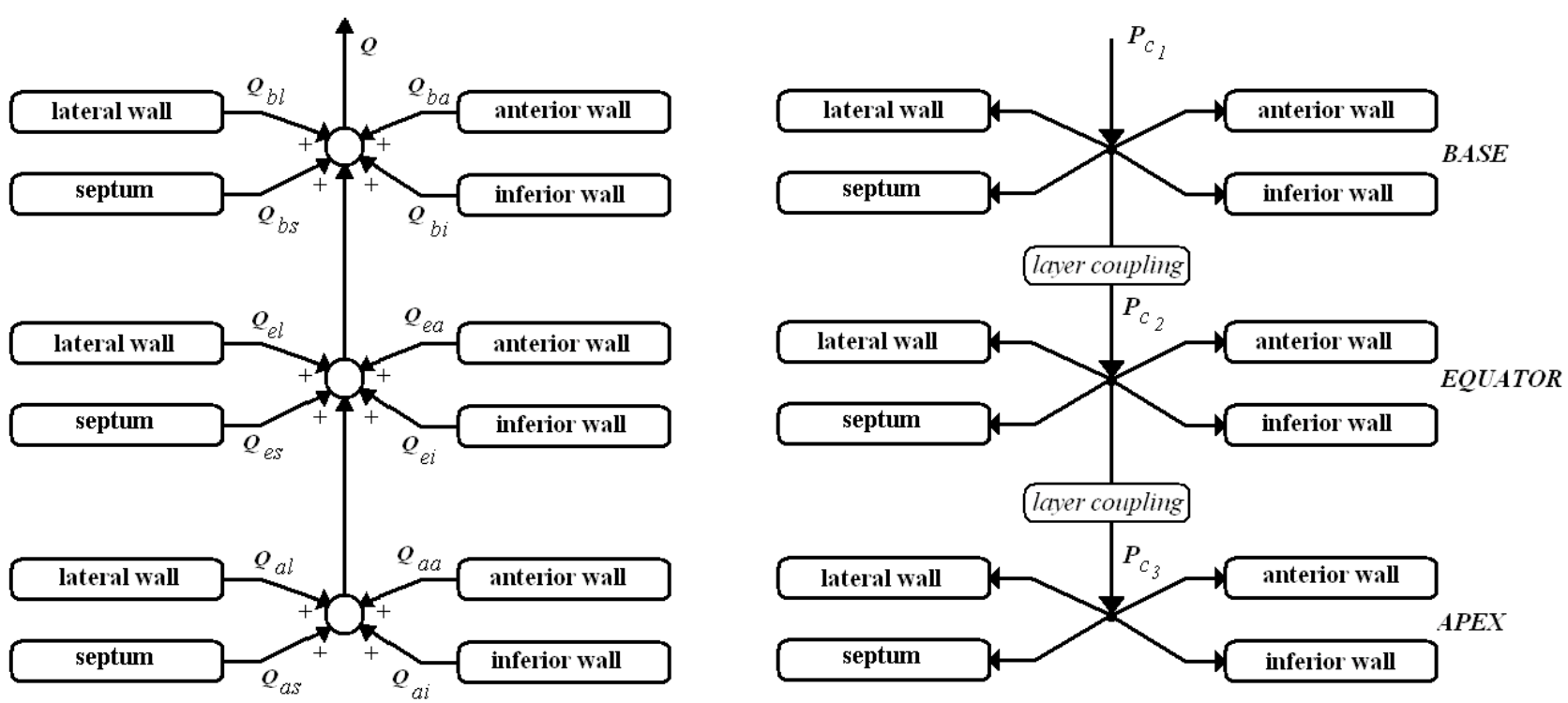

Figure 5: The twelve segments are connected in the hydraulic domain by adding each segment's flow and by supposing that the pressures are equal at the center of each layer.

The preload has been modeled by a constant flow source connected to a time-varying elastance that describes the atrial behavior [54]:

$$
E_{a}=\left\{\begin{array}{cc}
\frac{E_{\max }-E_{\min }}{2}\left(1-\cos \left(\frac{\pi \cdot t_{e a}}{T_{\max }}\right)\right)+E_{\min } & 0 \leq t_{e a} \leq 2 T_{\max } \\
E_{\min } & 2 T_{\max } \leq t_{e a} \leq T
\end{array}\right.
$$

where $t_{e a}$ corresponds to the time elapsed since atrial electrical activation. The afterload is described by a Windkessel model composed of a capacity, a resistance and an inertance. The heart valves are represented by non-ideal diodes that correspond to modulated resistances and the valvular plane is described by a linear capacitance.

In summary, the ventricular model is based on an ellipsoidal geometry and is composed of twelve segments which describe the different energy domains involved in cardiac function: i) the electrical activity is described by a cellular automata network, ii) the mechanical-hydraulic model represents the influence of both active and passive forces developed by each segment on the blood inside the cardiac cavity. The twelve segments are differentiated by specific parameters characterizing their electrical and mechano-hydraulic properties.

The values for most of the parameters describing the mechanical behavior $(c 1, c 2, c 3, c 4, c 5$, Tref, Bo, B1, nref, pC50ref, B2) have been taken from other studies [25, 52]. The parameter values controlling afterload and preload (capacitance, resistance, inertance and elastance) have been taken from [54, 55]. Appendix B presents all these parameter values. The other parameters values are determined by means of the identification algorithm. These parameters include the mechanical activation period $\left(T_{\max }\right)$, the maximum activation level $(K)$ and the $R D P$ period, for each segment.

Figure 6 presents the simulation of overall hemodynamic variables: ventricular pressure, flow and volume 
i58 for a normal cardiac cycle. Parameters for cellular automata have been fixed to fit electrical activation i59 patterns from [51]. The activation duration is equal to $400 \mathrm{~ms}$ [26] and the maximum level of calcium :60 concentration is fixed at $7 \mu \mathrm{M}[27]$.
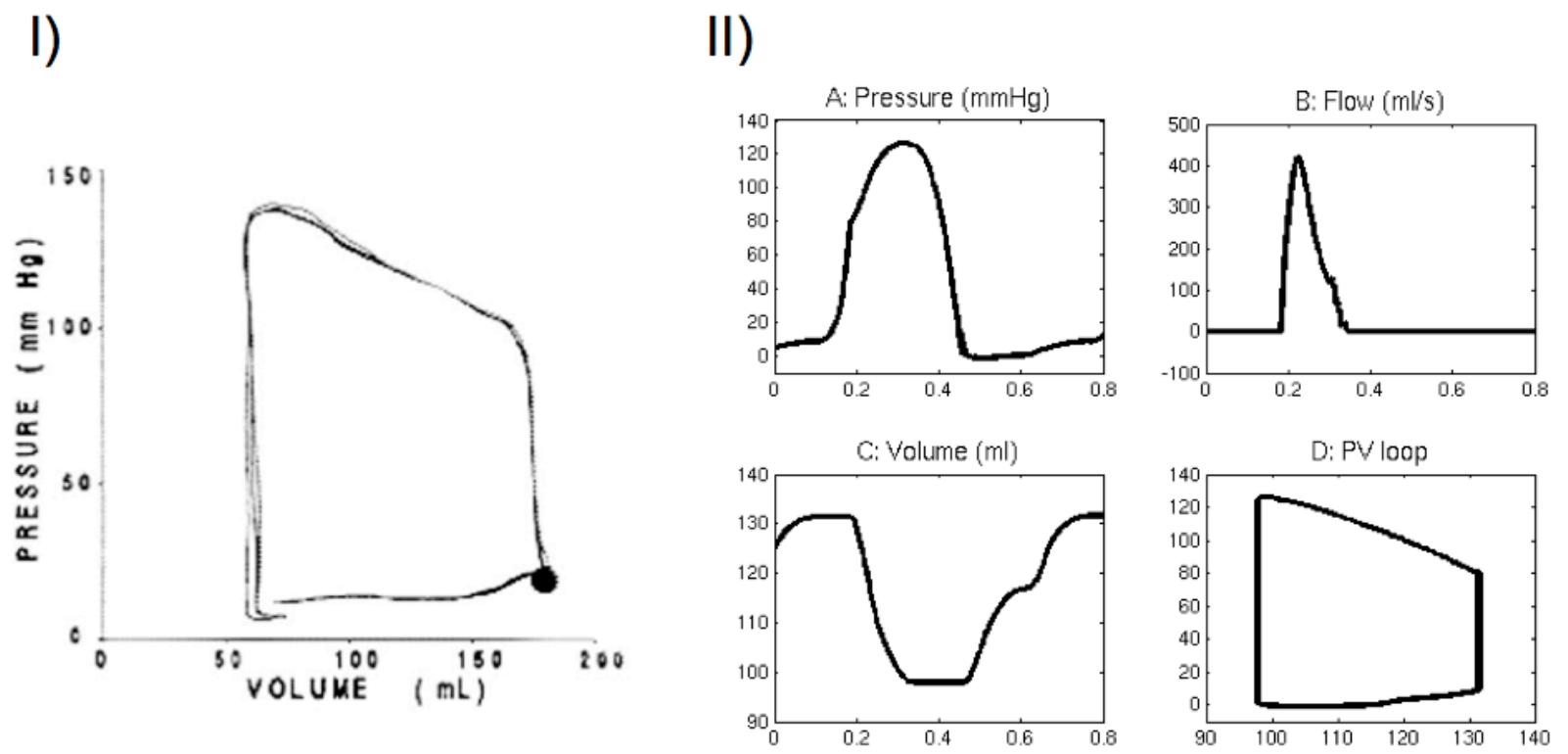

Figure 6: I) Stable resting left ventricular pressure-volume loop acquired from a human. The figure has been reproduced with permission from [65], II) Model simulation of ventricular hemodynamic variables for one beat: $A$ ) intra-ventricular pressure $B$ ) aortic flow $C$ ) volume $D$ ) pressure-volume loop.

;66 Figure 7 shows simulated strains in basal and equatorial segments for a normal and a pathologic case. The $: 67$ simulation of one cardiac cycle ( $800 \mathrm{~ms}$ of simulation), takes about 20s on a dual-core Intel Xeon 2.66Ghz.

;69 The normal case (figure 7A) is simulated by using the same parameters as those used in figure 6. A pathologic condition has been simulated by applying an additional electrical activation delay of $50 \mathrm{~ms}$ to the anterior basal segment (figure 7B). The consequences of this regional desynchronisation can be observed on the simulated strains during the isovolumic contraction phase, as the normal activation of the other myocardial segments has produced an extension of the delayed segment. This phenomenon is marked with a circle in figure 7B. 


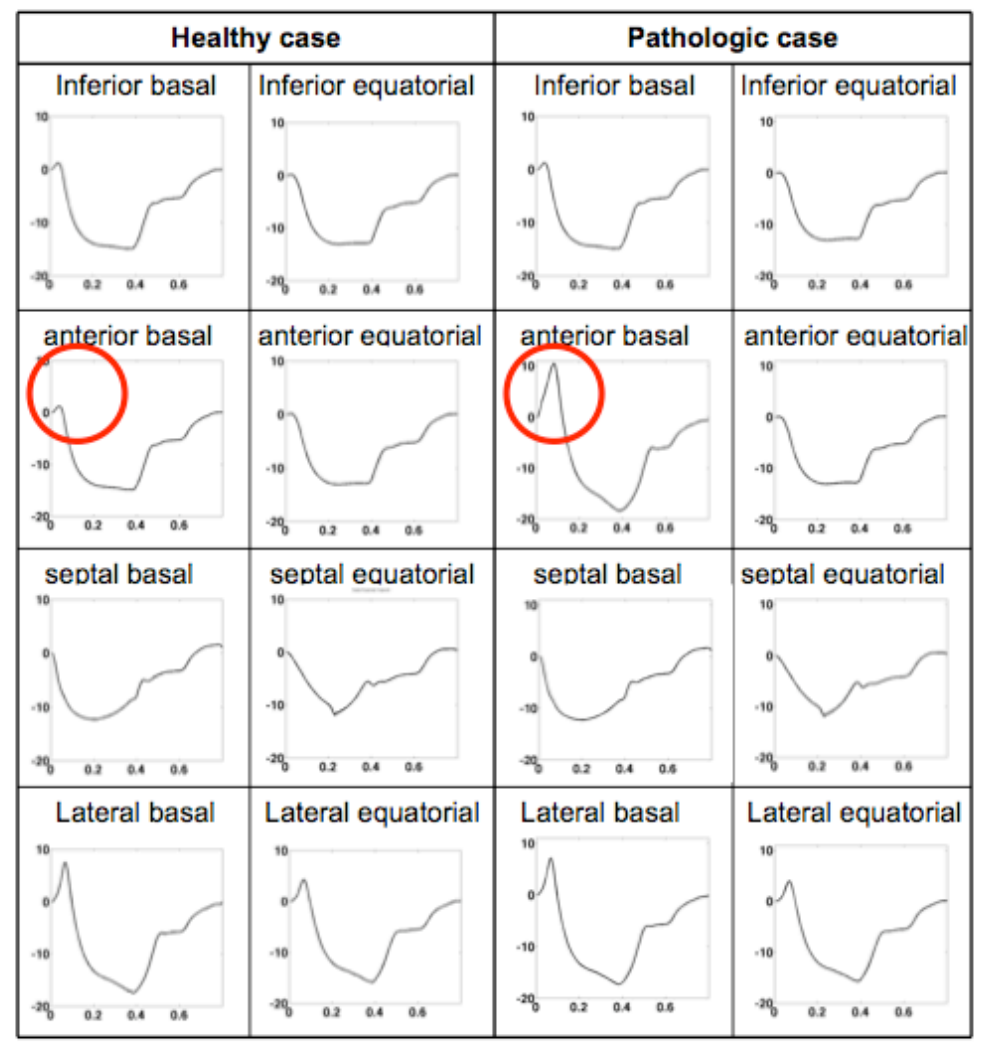

Figure 7: Strain simulation in healthy (left panel) and pathologic (right panel) conditions. The pathologic case was simulated by applying an electrical activation delay of $50 \mathrm{~ms}$ on the anterior basal segment. The extension of the delayed segment can be observed for the pathologic case during the isovolumic contraction phase (marked with a circle).

\subsection{Model adaptation to experimental data}

:81 The model-based process, which is applied to the interpretation of strain morphology, is presented in figure i82 8. The whole process is composed of three main steps: i) acquisition of strain signals measured by TDI, ii) i83 adaptation of the model's geometrical shape to real dimensions, determined by echocardiography and the :84 identification of patient-specific parameter values to reproduce the observed strain and iii) the physiological :85 interpretation of the identified parameters. This model-based approach is based on studies completed by our :86 laboratory which led to patient-specific parameter identification methods with applications in cardiology [50] :87 or epileptology [56]. 


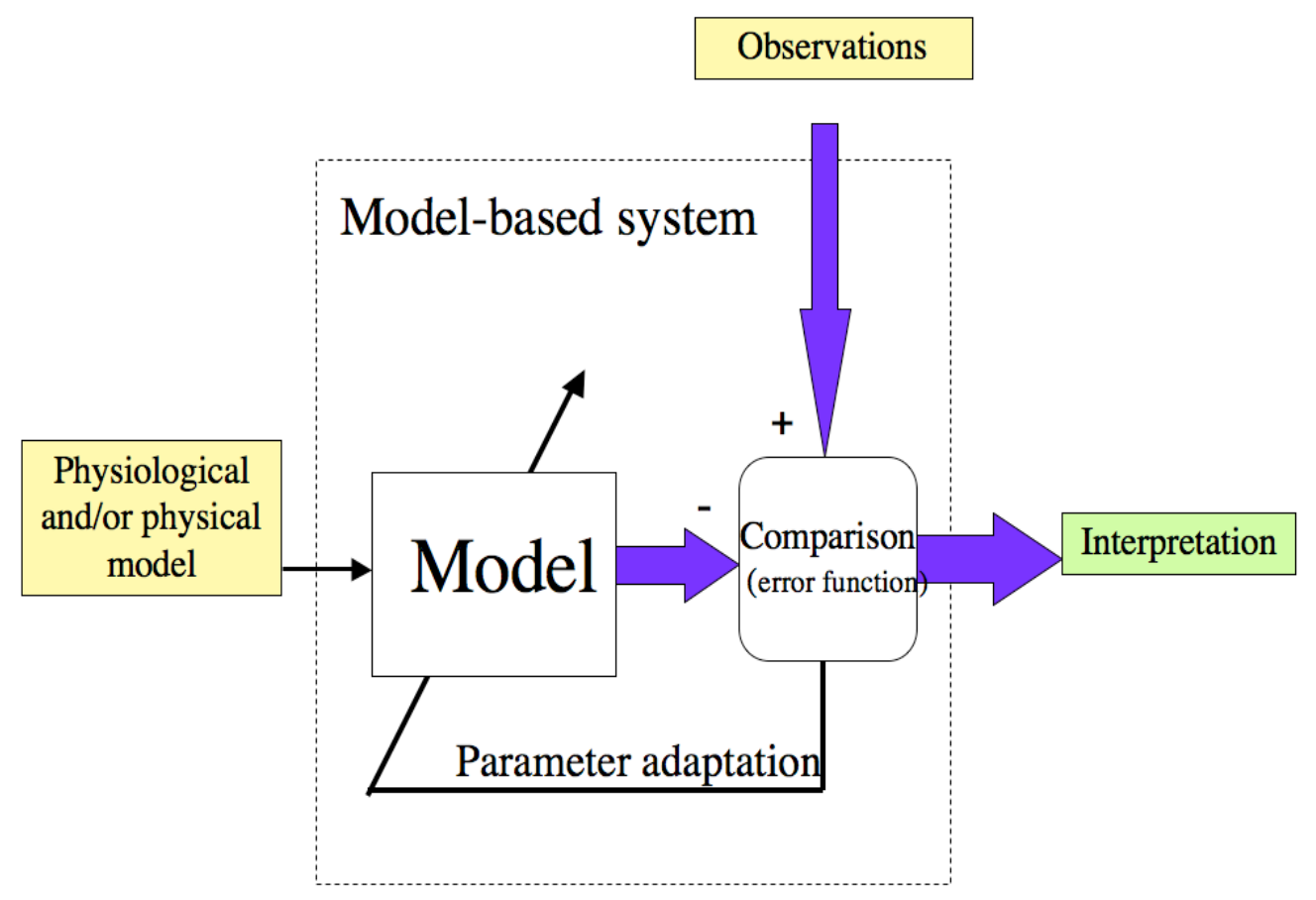

Figure 8: Parameters adaptation process. The evaluation of the error between observable signals and simulations is minimized in order to determine the model's parameters using an identification algorithm. The parameter values are representative of the physiological system state and can help to interpret observations.

993 Concerning the adaptation of the geometrical shape, echocardiography reports usually inform us of the i94 ventricle's length $(L)$ and diameter $(D)$. The model's ellipsoid dimensions are defined from this data. In fact, i95 if the diameter is supposed to be measured at the equator, the minor axis can be computed as $a=D / 2$. i96 Additionally, a relation between the major axis and the ventricle length is defined in [57] as $b=2 L / 3$ (figure $: 97$ 9). Since the major and minor axes are known, it is possible to calculate, for each segment, the surface $(S)$ $i 98$ and the radii of curvature in order to get coefficients $K_{\theta}, K_{\varphi}, K_{r}$. 

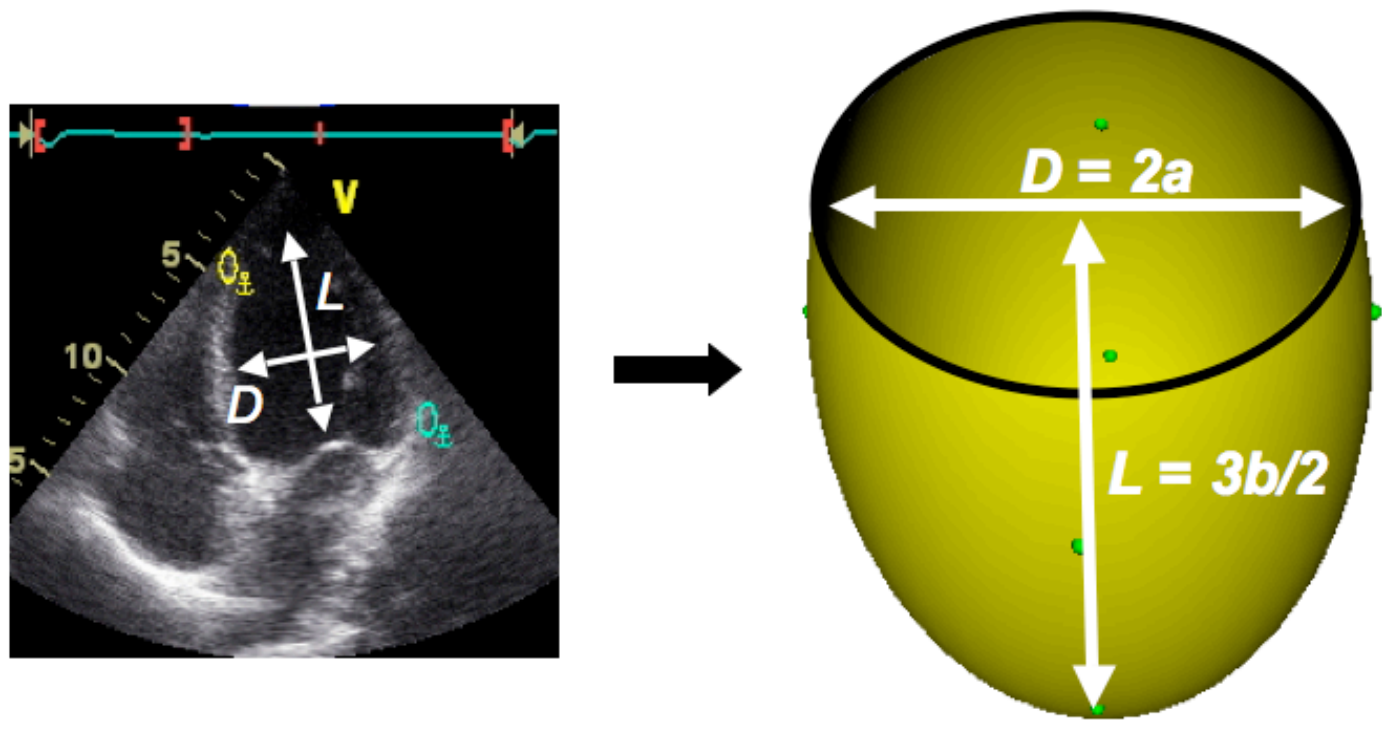

Figure 9: Ellipsoidal model dimensions obtained from echocardiographic measures: a and b are respectively the minor and major axis sizes.

The identification algorithm is used to minimize the difference between experimental and simulated strains on the eight myocardial segments at the base and at the equator. Parameters related to the four apical segments have not been identified, as the strain data from these segments are difficult to acquire. For these apical segments, the model parameters have been fixed from mean physiological values. These values are listed in appendix 2 .

\subsubsection{Identification algorithm}

The parameter identification can be seen as an optimization problem consisting of minimizing, for each beat $i$, an error function defined as the difference between the synthesized strains and the observed strains. The synthesized strains are obtained by simulating the proposed model $M$ with a specific set of parameter values $P$, such that:

$$
\begin{gathered}
X_{s}^{o b s}=\operatorname{Strain}_{s}^{o b s}(t) \\
X_{s}^{\text {sim }}=\operatorname{Strain}_{s}^{\text {sim }}(t)=M(P)
\end{gathered}
$$

where $X_{s}^{\text {obs }}$ and $X_{s}^{\text {sim }}$ represent, respectively, the observed and simulated strains for segment $s$ and $t=\left[\tau_{Q R S_{i}}, \ldots, \tau_{Q R S_{i+1}}\right]$ where $\tau_{Q R S_{i}}$ represents the QRS detection instant for beat $i$.

The parameter set $P$ defines the following values for each segment: the mechanical activation period $\left(T_{\max }\right)$, the maximum activation level $(K)$ and $R D P$ period ( $\left[R D P P_{-}\right.$segment, $K_{-}$segment,$T_{-}$segment $\left.]\right)$. Additionally, two hydraulic resistance values are determined at the base and the equator ( $\left[R_{-}\right.$max $_{-}$base, $R_{-}{ }_{\text {min_base, }} R_{-}$max_equateur, $R_{-}$min_equateur $\left.]\right)$. In total, 28 parameters have to be identified and $P$ is defined as $P=\left[R_{-}\right.$max_base, $R_{-}$min_base, $R_{-}$max_equateur, $R_{-}$min_equateur, $R D P_{-}$ant_base, $R D P_{-}$inf_base, $R D P P_{-}$lat_base, $R D P_{-}$septum_base, $R D P_{-}$ant_equa,

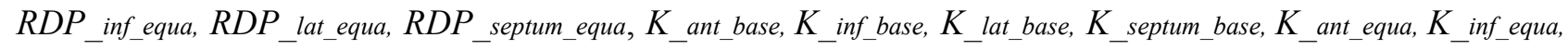

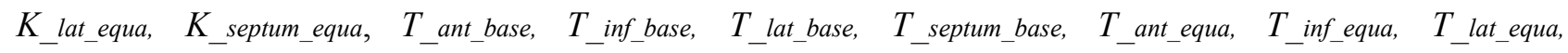
$T_{-}$septum_equa]. The objective is thus to obtain an optimal set of patient-specific parameters $P^{*}$ which 
minimize an error function between $X_{s}^{\text {obs }}$ and $X_{s}^{\text {sim }}$. This error function has been defined here as the sum of

t25 the absolute values of the difference between each experimental and simulated strain, calculated for the t26 whole cardiac cycle and for the 8 basal and equatorial segments.

$$
\varepsilon=\sum_{s=1}^{8} \sum_{t=\tau_{Q R S_{i}}}^{\tau_{Q R S_{i}+1}}\left|\operatorname{Strain}_{s}^{\text {sim }}(t)-\operatorname{Strain}_{s}^{o b s}(t)\right|
$$

This error function is not differentiable and can have multiple local optima. This kind of problem can be solved using Evolutionary Algorithms (EA), which are an adapted method used in identifying complex nonlinear problems characterized by a poorly-known state-space. EA are stochastic search techniques, inspired by the theories of evolution and natural selection, which can be employed to find an optimal configuration for a given system within specific constraints [58].

.33 In these algorithms, the set of parameters $P$ characterizes each "individual" of a "population". In order to .34 reduce the search space, values for parameters were bounded to the physiologically plausible intervals: [0.5 .35 5] for the maximum hydraulic resistances, [ $\left.\begin{array}{lll}0.01 & 0.5\end{array}\right]$ for the minimum hydraulic resistances, [0.3 0.9$]$ for t36 calcium period, [5 12] for calcium amplitude and [1 500] for electrical time activation. These intervals have 137 been defined around parameter values used for the simulation of global hemodynamic variables in section .38 3.1.5 (taken from other studies) and are based on physiological knowledge on the electromechanical 139 activities of the heart.

40 An initial population is created from a set of randomly generated individuals. The 28 parameter values of a 41 given individual are independently generated from a uniform distribution, defined under the corresponding 142 feasibility interval. This population will "evolve", minimizing the error function, by means of an iterative 143 process (figure 10).

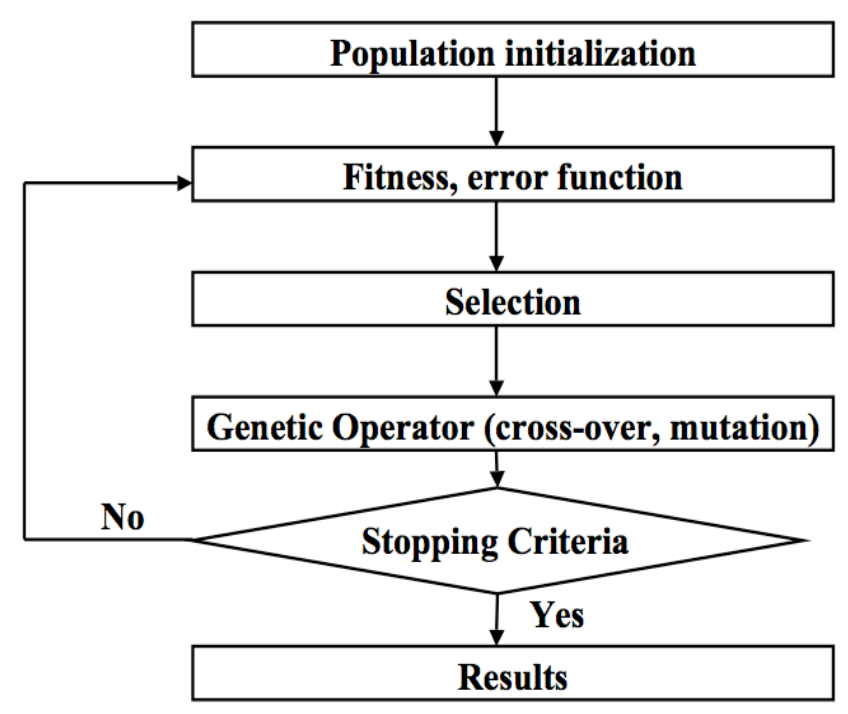


Once the error function has been evaluated for each individual, a new generation is produced by applying mutation and crossover operators on selected individuals. The selection is carried out by means of the "roulette wheel" method, adapted for function minimization, in which the probability of selecting a given individual depends on the value of its error function, divided by the sum of all the error values of the population. Only standard genetic operators, defined for real-valued chromosomes, have been used in this work: "uniform crossover", which creates two new individuals (offspring) from two existing individuals (parents), by randomly copying each allele from one parent or the other, depending on a uniform random variable and "Gaussian mutation", which creates a new individual by randomly changing the value of one allele (selected randomly), based on a Gaussian distribution around the current value. More details on these kinds of optimization methods can be found on [59-61].

\section{Results and Discussion}

\subsection{Acquisition protocol}

Strain acquisition using color tissue Doppler imaging has been applied to two healthy subjects and one patient affected with dilated cardiomyopathy (DCM). This pathology is characterized by a heart enlargement and a reduced mechanical cardiac function. Ultrasound measurements were performed in order to determine the LV dimensions on two cardiac cycles.

For the two healthy subjects and the pathologic patient, the minor (a) and major (b) axes values are first determined from echocardiography (Erreur! Source du renvoi introuvable.). Dimensions for the pathologic patient are particularly high, which is coherent with the diagnosis of a DCM.

\begin{tabular}{|lccc|}
\hline & Healthy & Healthy & DCM \\
\hline minor axis $\boldsymbol{a}$ & 1.9725 & 1.8575 & 3.22 \\
\hline major axis $\boldsymbol{b}$ & 4.8067 & 4.8033 & 5.99 \\
\hline
\end{tabular}

Table 1: Minor (a) and major (b) axis values for the three patients.

\subsection{Comparison between simulated and experimental strain}

Strain signals have been measured on eight segments of the myocardial wall at the base level (the septum and the anterior, inferior and lateral wall) and the equatorial level (the septum and the anterior, inferior and lateral wall). In fact, it is not possible to obtain accurate data on the apical segments using TDI. Figure 11 shows simulations obtained after parameter adaptation and the clinically recorded strain signals for the three subjects under study. The mean error value (calculated on the eight segments as presented in equation 21) is equal to $2.4 \%, 2.09 \%$ and $1.30 \%$ respectively for the two healthy subjects and the pathologic patient, despite the important disparity on the data from each subject. 


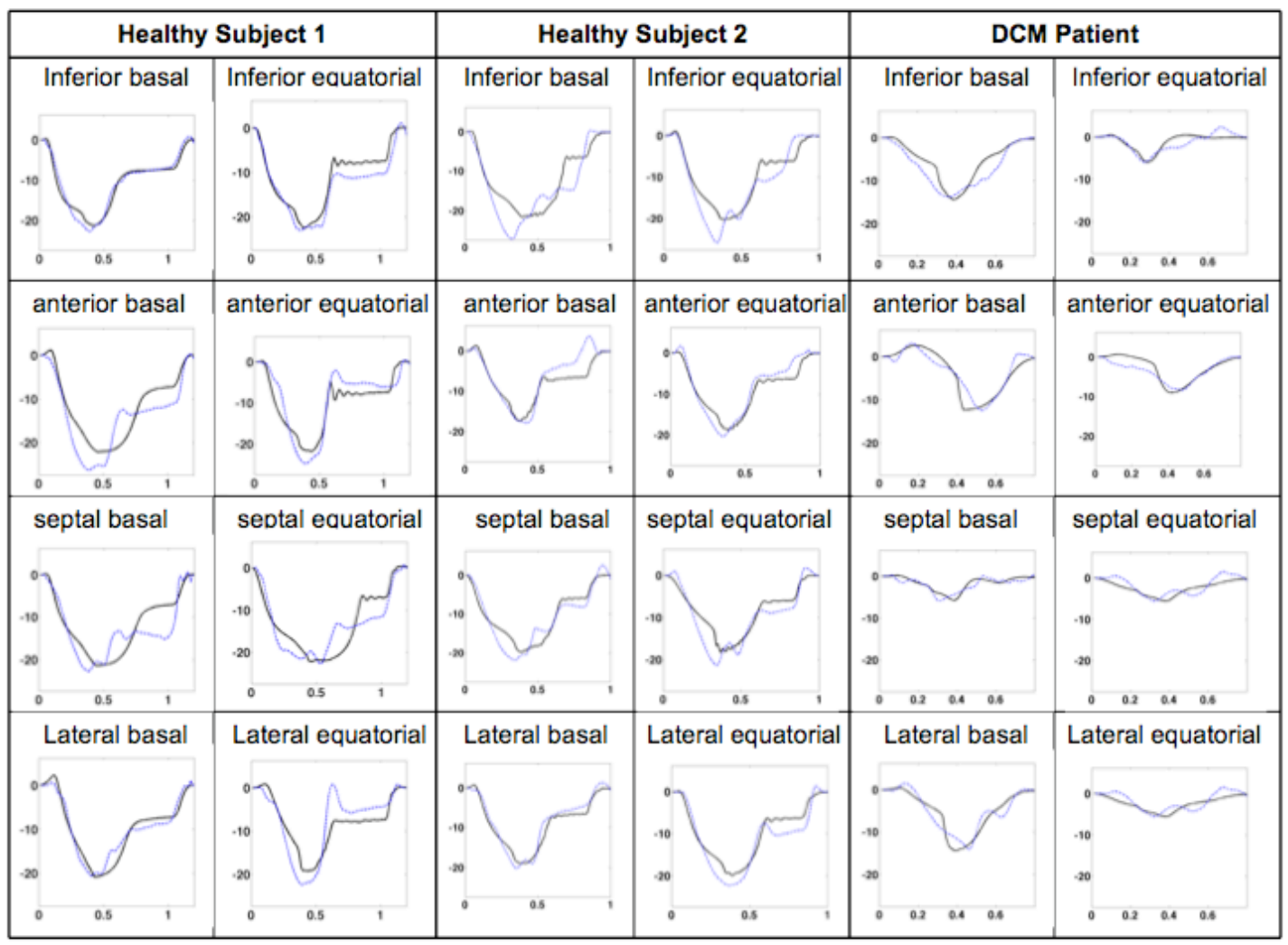

Figure 11: Comparison between simulated and experimental strains for the two healthy subjects and the pathologic patient during one cardiac cycle for the septum, anterior, inferior and lateral walls of the base and equator. The continuous curve is the simulation and the segmented one is the experimental data. The strain is expressed as a percentage of the diastolic state.

183 Simulated systolic peak times are coherent with the observation and the reproduction of the overall 184 morphology of the isovolumic contraction and systolic phases is approached with relative errors of $2.12 \%$, $1852.16 \%$ and $1.25 \%$ for the first, second and third subject, respectively. Additionally, the simulations reproduce some particularities that are due to the pathology. In fact, for the patient suffering from DCM, positive values on some strain signals can be observed during the isovolumic contraction. These strain elevations are due to increased electrical activation delays in the corresponding segments and they can be explained by the extension of yet inactivated segments during the contraction of the other segments. This example shows how the model-based approach may assist in the interpretation of myocardial strain morphologies.

\subsection{Interpretation of the identified parameters}

193 The previous results can be represented graphically by visualizing the deformations which have been applied 194 to the twelve myocardial segments at each time step. Simulated strains, obtained using patient-specific parameters, are applied at the center of the corresponding myocardial segment. The cardiac surface deformation is computed using Thin Plate Spline (TPS), which is a generalization of 1-D cubic splines [62]. 
197 The surface is represented by a graph on which are defined landmarks (data points). The position of each 198 landmark being known during the whole cardiac cycle, the cardiac surface deformation is computed using 199 TPS interpolation (based on a bending energy, defined as the integral, over all the mesh nodes, of the squares ;00 of the second derivatives). Figure 12 shows the ventricular deformation and the identified electrical activity ;01 for the three subjects during the cardiac cycle. The electrical activation sequences for the two healthy ;02 subjects are coherent with well-known activation maps [51]. It possible to observe a reduced mechanical activity and a delayed electrical activation on the DCM patient. A qualitative evaluation of the three ;04 simulated sequences has been performed by a cardiologist. He confirms that simulations correspond to pathophysiologically plausible sequences. The delayed electrical activity observed in the simulation for the i06 DCM patient can be explained by an intra-ventricular desynchronisation, frequently observed in this kind of pathology. A quantitative evaluation of the estimated activation sequence could be performed by acquiring an electrophysiological cartography for each patient. This delicate invasive procedure has not been applied for the patient analyzed in this paper. However, this kind of evaluation will be possible using data acquired on a current clinical protocol running at the Rennes University Hospital.

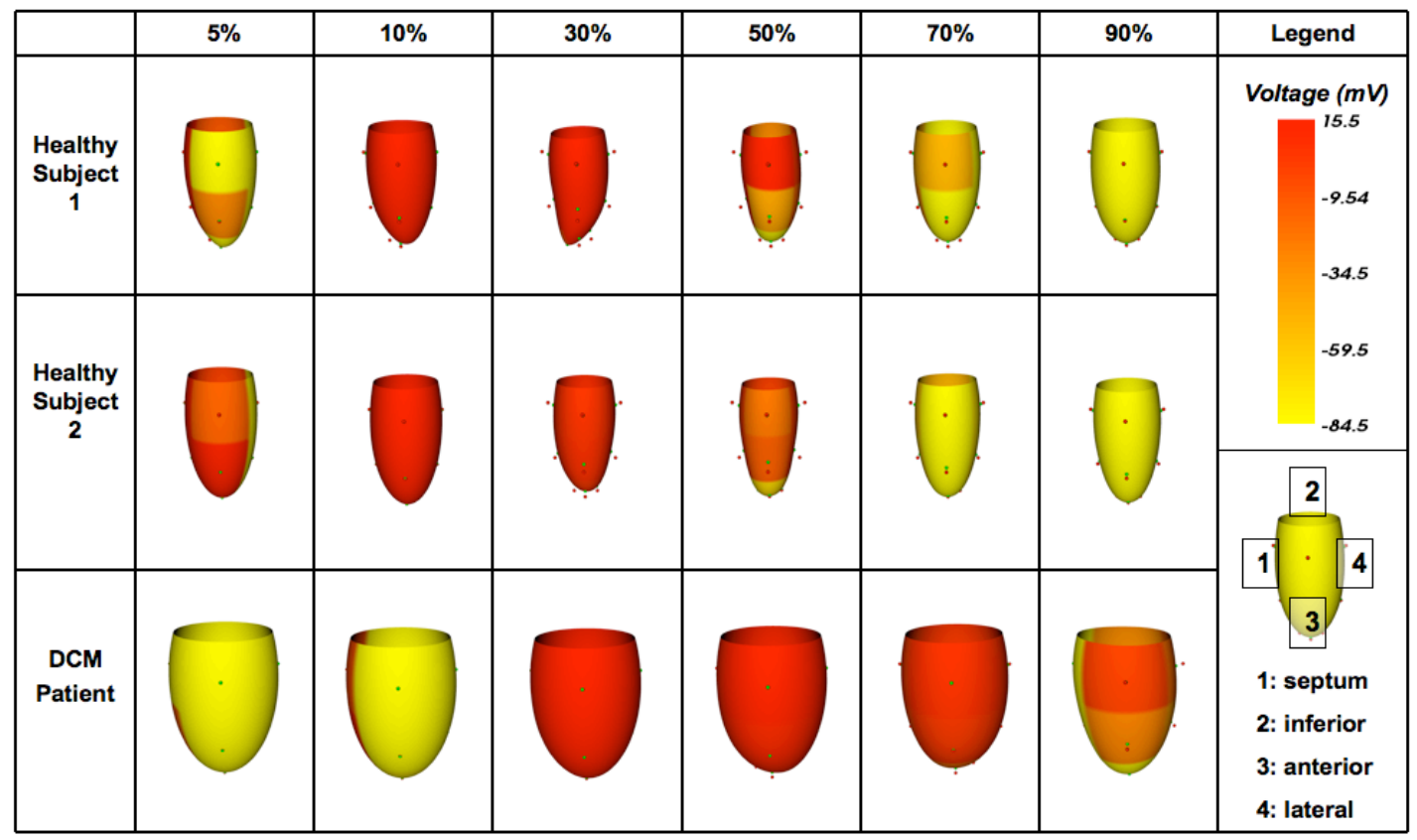

Figure 12: Contraction of the electromechanical model for the two healthy subjects and the pathologic patient. The color legend corresponds to the electrical state of each segment and is expressed in $\mathrm{mV}$. The orientation of the ventricular model is depicted in the lower right panel of the figure.

The identification process brings patient-specific parameters that are interesting to analyze, since they can be representative of the physiopathological state. To facilitate the visualization of these results, the "Bull's eye" representation is used to depict the electro-mechanical parameters identified for each segment (figure 13). 


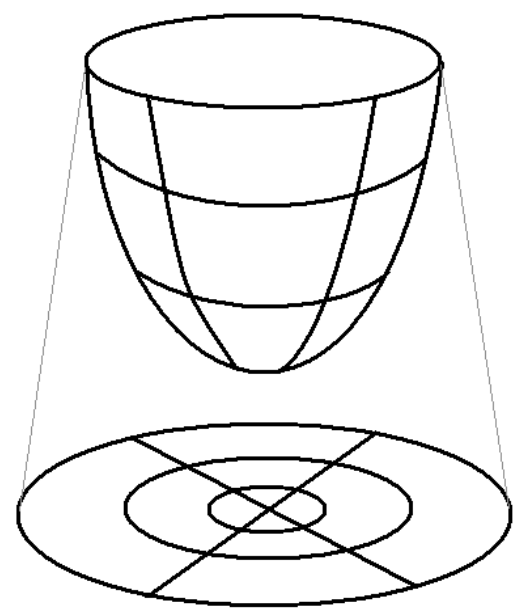

\begin{abstract}
Figure 13: Bull's eye representation of ventricular parameters. For each Bull's eye diagram, the left, top, right and down parts represent the septal, anterior free wall and posterior regions, respectively. The outer, mid- and inner rings represent the base, the equator and the apex of the LV. This representation will be used in figures 14 and 15.
\end{abstract}

Figure 14 shows the electrical activation times for the three patients. The maximum values reach $54 \mathrm{~ms}, 67.4$ $\mathrm{ms}$ and $126 \mathrm{~ms}$ for the first and second subjects and the DCM patient, respectively. It is easy to verify that the pathologic patient presents increased electrical activation delays in comparison with well-known activation maps [51].

The mechanical activity peak time $T_{M P}$ can also be analyzed. In order to compare this parameter for the three patients, the identified $T_{M P}$ is expressed as a percentage of the cardiac cycle duration ( $R R$ interval):

$$
T_{M P \%}=100 . T_{M P} / R R
$$

Figure 15 shows the bull's eye representation of identification results. For the first healthy subject, the mechanical activity peak time goes from $18 \%$ to $37 \%$ of the RR interval. For the second one, this parameter varies from $21 \%$ to $37 \%$. $T_{M P \%}$ ranges from $31 \%$ to $60 \%$ for the affected patient.

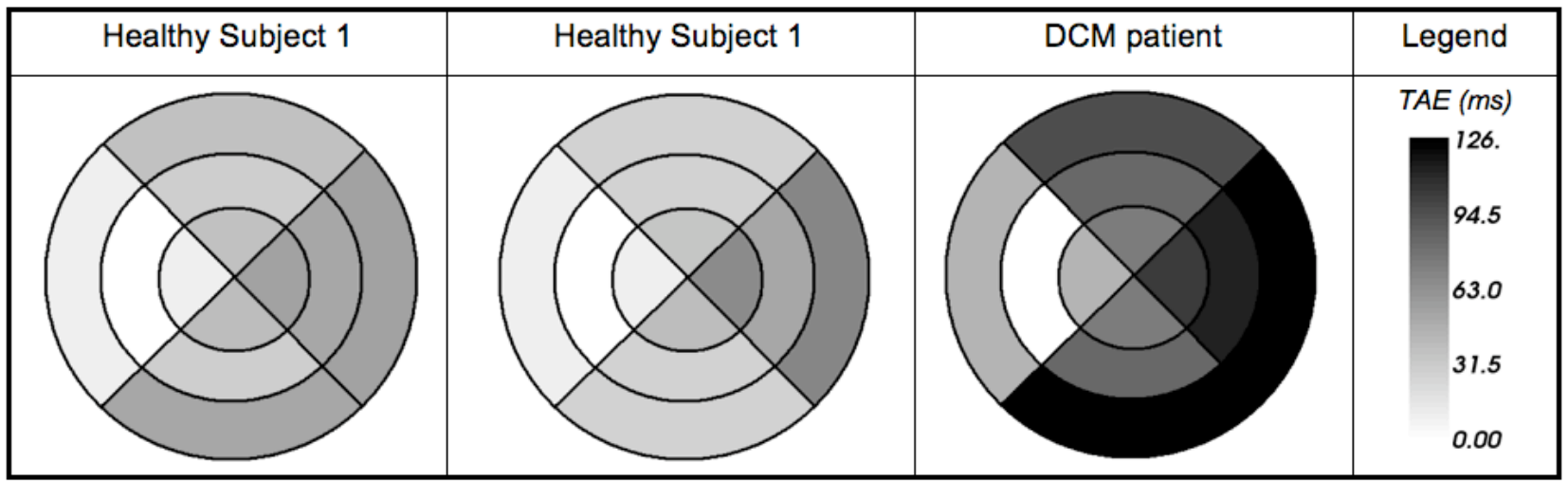

Figure 14: Bull's eye representation of the electrical activation times (TAE) for the two healthy subjects and the pathologic patient. The 
The $T_{M P \%}$ comparison for both healthy subjects shows similar value ranges. The third patient percentages are higher overall. These values are particularly high for the anterior and lateral segments of the base because they are respectively equal to $60 \%$ and $55 \%$. The increased $T_{M P \%}$ is a marker of a myocardial dysfunction concerning these two segments. The model-based localization of the delayed segments and the magnitude of the desynchronization for the DCM patient are in accordance with the diagnosis provided by the expert cardiologist. Moreover, the model-based approach provided further insight in the explanation of the observed strain morphologies.

\begin{tabular}{|l|l|l|}
\hline Healthy Subject 1 & DCM patient & Legend \\
\hline Healthy Subject 2 & TMP\% \\
\hline 39.0
\end{tabular}

Figure 15: Bull's eye representation of the mechanical activity peak time expressed as a percentage of the RR-interval (TMP\%) for the two healthy subjects and the pathologic patient. These values are high for the anterior and lateral segments of the DCM patient.

\subsection{Robustness analysis}

In order to test the robustness of the identification method, the algorithm has been repeated ten times on one subject. Figure 16 shows boxplots of the identified $T_{M P}$ parameters. The results of the ten identifications are

i52 close enough to show that the parameter interpretation is available from a physiological point of view.

i53 Furthermore, it can be seen from these box-plots, and from results in section 4.3, that the identified i54 parameter values do not reach the upper or lower boundaries of the physiological plausible intervals defined i55 in section 3.2.1. 


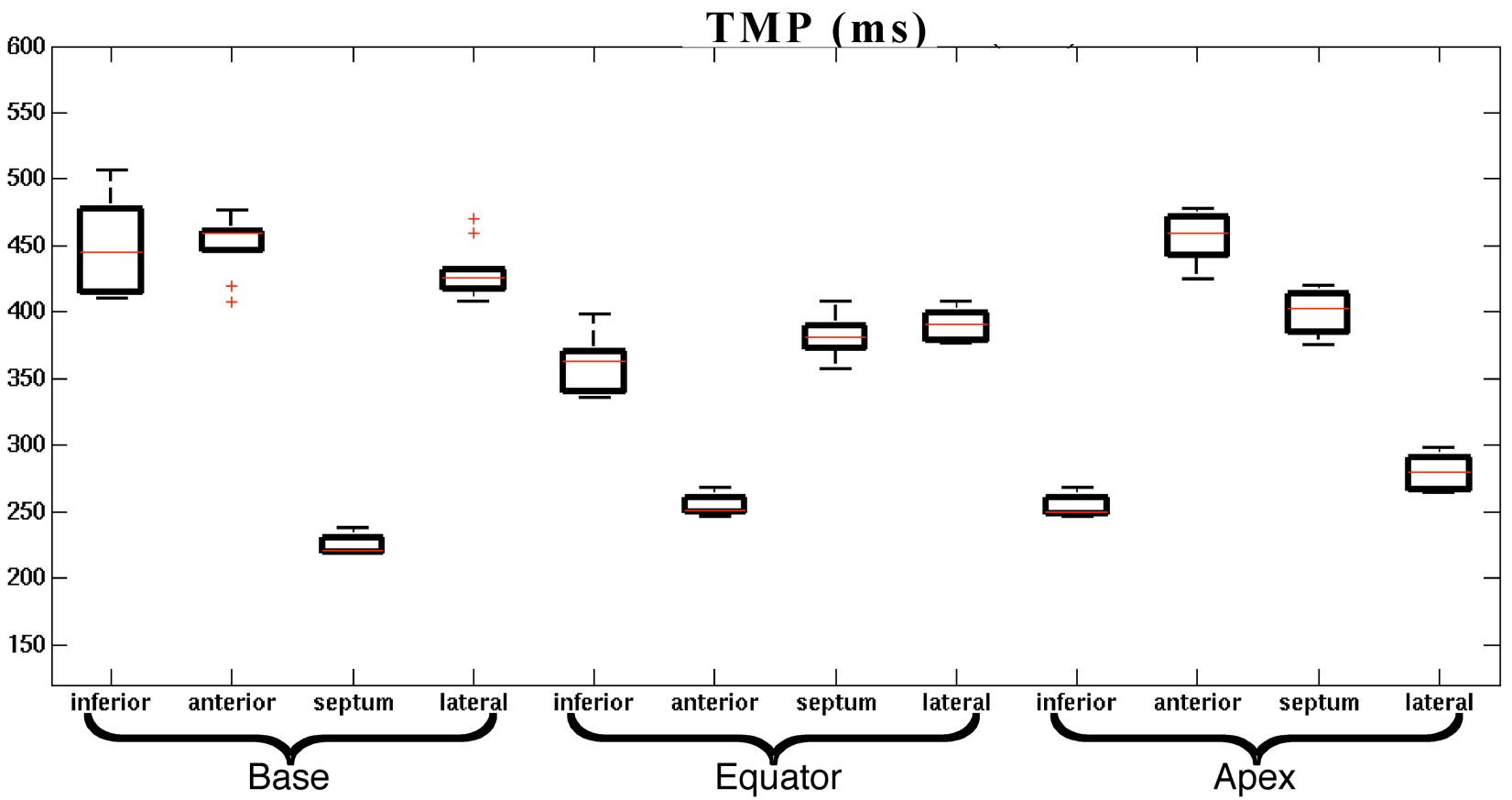

Figure 16: The identification algorithm has been repeated ten times on one subject in order to test the robustness of the identified parameters. This figure shows the boxplot of the ten identified $T_{M P}$ for the first healthy subject.

\subsection{Study Limitations}

The main limitations of the proposed approach are related to the hypotheses made to build the ventricular model, the observability of TDI strain analysis, and the current clinical evaluation state.

;62 Concerning the ventricular model, the following hypotheses were made in order to obtain a tissue-level, lightweight model on which we could perform parameter identification from observed strain signals: $i$ ) the ventricular torsion motion has been neglected, ii) the mechanical continuity between segments is not always assured, as the ventricle is represented by a set of sub-pumps coupled in the hydraulic domain and commanded by a coordinated electrical activity, iii) the intracellular calcium concentration is approximated by an analytic expression and $i v$ ) the myocardial fiber orientation is assimilated to a mean angle. Although these simplifications have a direct consequence on the synthesis of strain signals, in particular during the diastolic period (as discussed above), the authors consider that they are in accordance to the problem under study and the resulting model can already be useful to assist in the interpretation of strain data. Another limitation concerns the limited observability of the apical segments from strain analyses. As already mentioned, in order to overcome this problem, the model parameters for the apical segments have been fixed from mean physiological values and are not included into the identification process.

Finally, concerning the clinical evaluation of the approach, only a limited number of healthy subjects and DCM patients have been analyzed so far. Although these results are encouraging and show the feasibility of the approach, a larger clinical evaluation is necessary in order to validate the interpretations obtained from this model-based approach. 


\section{Conclusion}

;80 The present paper described a model-based approach for the analysis of myocardial strain data. The method ;81 is based on a tissue-level model of the left ventricle that includes a simplified geometrical representation, a i82 description of the electrical, mechanical and hydraulic activities and a physiological segmentation, which are i83 in accordance to the problem under study. As low computational resources are required for the simulations i84 of the proposed model, the identification of patient-specific parameters becomes feasible.

;85 Patient-specific parameters were obtained for two normal subjects and one patient suffering from DCM. The ;86 mean error between observed and synthesized strain signals, after parameter identification, is particularly ;87 low (between 1.30\% and 2.34\%). A qualitative comparison between the results obtained from the proposed ;88 model-based approach and a classical TDI analysis has been performed by a cardiologist/echocardiographer i89 (ED). The localization of failing segments and the values and instants of occurrence of the peak systolic ;90 strains have shown to be coherent with the clinical analysis. The model also allows a better reproducibility in ;91 the estimation of systolic peaks and adds the possibility of estimating electrical activation times, which are ;92 difficult to observe experimentally. This appears particularly useful as echocardiographic strain signals are ;93 sometimes noisy and challenging to interpret, limiting its large use in routine clinical practice. Recent papers ;94 insist on this problem of reproducibility and robustness of the echocardiographic interpretation (PROSPECT ;95 study [63] and RETHINQ study [64]).

;96 Moreover, such a patient-specific model-based method can be used to assist the interpretation of strain ;97 morphologies and to find, via simulations, the origin of particular strain shapes. The main advantage of this ;98 model-based approach with respect to "black-box" approaches, such as neural networks, is that the patienti99 specific parameters characterizing the model provide a direct physiological interpretation. Due to this i00 property, these model-based approaches can be useful for a better interpretation of the morphology of strain i01 signals and to assist diagnosis and therapy definition.

;02 Current work is directed towards the improvement of the ventricular model and a further clinical evaluation i03 of the proposed approach. In this sense, a clinical protocol including electrophysiological cartography, TDI i04 and multi-slice computer tomography on patients receiving cardiac resynchronization therapy is currently in i05 progress in the Rennes University Hospital (IMOP project).

\section{i06 Acknowledgements}

;07 The authors would like to thank Antoine Simon for his valuable assistance in the exploitation of the i08 visualization tools.

\section{Appendix A}

i10 The strain $\varepsilon$ is a mechanical expression reflecting the percentage deformation of a given myocardial segment i11 with respect to its original end-diastolic state and can be defined as: 


$$
\varepsilon=\frac{l-L}{L}
$$

i13 where $l$ is the current length of the object and $L$ its original length. The main concepts of TDI acquisition and i14 analysis are explained in $[1,2]$. Briefly, the instantaneous change in length $(\mathrm{d} l)$ of a given object can be i15 obtained from the estimation of the velocity of two different points at the end extremity of the object of i16 interest ( $v 1$ and $v 2)$, as

$$
\mathrm{d} l=(v 1-v 2) \mathrm{d} t
$$

i18 This quantity can be divided by L in order to obtain the velocity gradient, or strain rate SR, which is thus i19 defined as:

$$
\frac{\mathrm{d} l}{l}=\frac{v 1-v 2}{l} \mathrm{~d} t
$$

i21 As it is difficult to obtain the velocity of two points at the extremities of the object, the velocity gradient is i22 estimated from two points separated by a fixed distance $(\Delta r)$ and equation 25 becomes

$$
\frac{\mathrm{d} l}{l} \approx \frac{v(r)-v(r+\Delta r)}{\Delta r} \mathrm{~d} t
$$

$i 24$ Equation 26 is integrated to obtain:

$$
\log \frac{l}{L}=\int_{t_{o}}^{t} S R \mathrm{~d} t
$$

where log is the natural logarithm. The expression of strain can be deduced from the last equation:

$$
\varepsilon=\exp \left(\int_{t_{o}}^{t} S R \mathrm{~d} t\right)-1
$$

Myocardial strain estimation from TDI measurements are performed in practice as follows: The operator manually identifies one to six regions of interest and the software will automatically define two sets of points spaced from 9 to $12 \mathrm{~mm}$ within the defined region of interest. The software will then compare the velocity vectors of these two sets of points during a cardiac cycle to calculate strain and strain rate. It is important to note that the strain acquisition depends on the ultrasound beam orientation and the axis of the left ventricle.

\section{Appendix B}

i34 Table 2 presents the model parameter values, extracted from other studies, which have been left fixed on the 135 model (not identified), as well as their sources. 


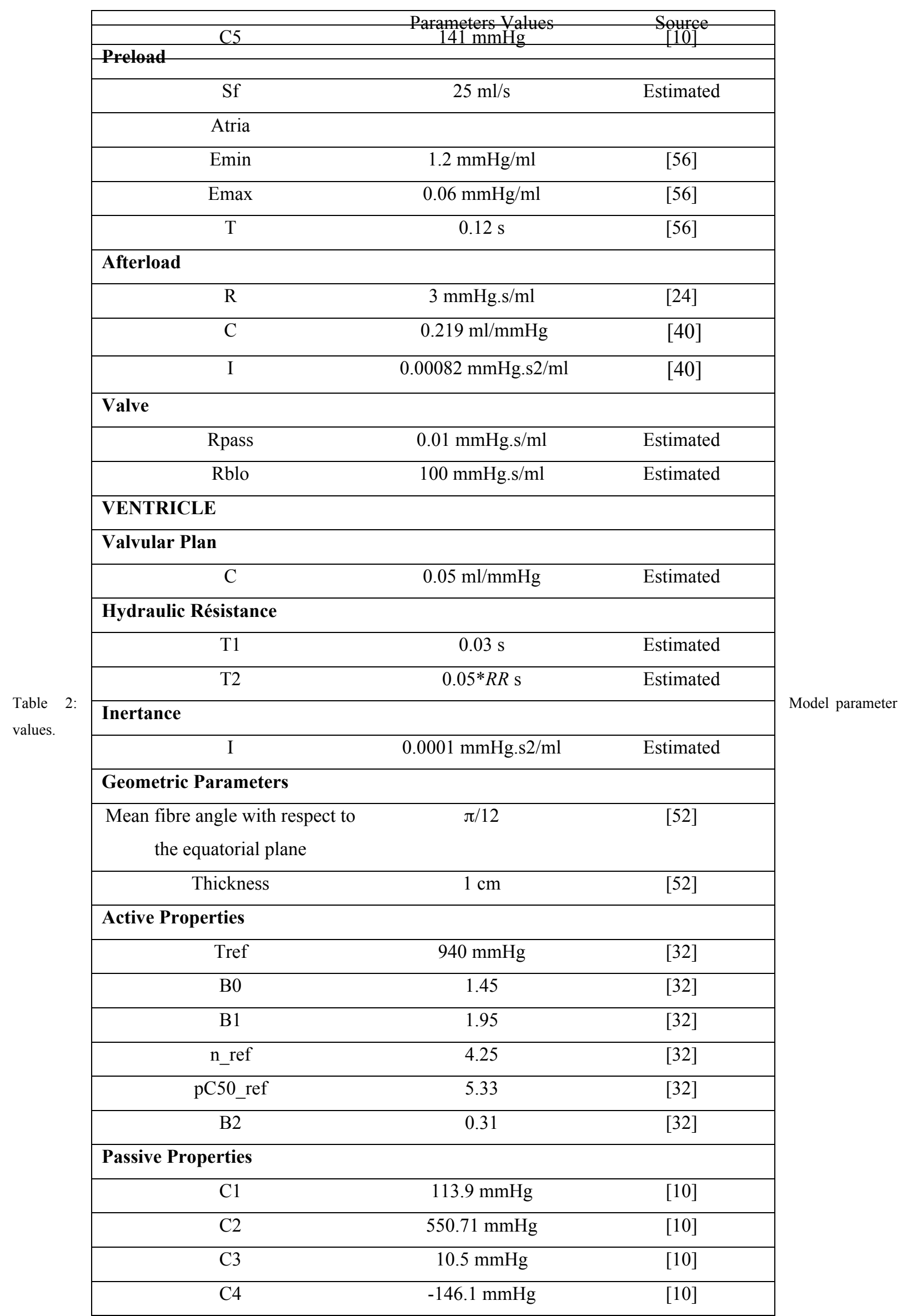




\section{References}

$i 61$

i62

[1] J. D'Hooge, A. Heimdal, F. Jamal, T. Kukulski, B. Bijnens, F. Rademakers, L. Hatle, P. Suetens, and G. R. Sutherland, "Regional strain and strain rate measurements by cardiac ultrasound: principles, implementation and limitations," European Journal of Echocardiography, vol. 1, pp. 154-70, 2000.

[2] S. Urheim, T. Edvardsen, H. Torp, B. Angelsen, and O. A. Smiseth, "Myocardial strain by Doppler echocardiography. Validation of a new method to quantify regional myocardial function," Circulation, vol. 102, pp. 1158-64, 2000.

[3] A. Heimdal, A. Stoylen, H. Torp, and T. Skjaerpe, "Real-time strain rate imaging of the left ventricle by ultrasound," Journal of the American Society of Echocardiography, vol. 11, pp. 1013-9, 1998.

[4] E. Donal, P. Raud-Raynier, D. Coisne, J. Allal, and D. Herpin, "Tissue Doppler echocardiographic quantification. Comparison to coronary angiography results in Acute Coronary Syndrome patients," Cardiovascular Ultrasound vol. 3, pp. 10, 2005.

[5] T. Kukulski, F. Jamal, L. Herbots, J. D'Hooge, B. Bijnens, L. Hatle, I. De Scheerder, and G. R. Sutherland, "Identification of acutely ischemic myocardium using ultrasonic strain measurements. A clinical study in patients undergoing coronary angioplasty," Journal of the American College of Cardiology, vol. 41, pp. 810-9, 2003.

[6] T. Edvardsen, B. L. Gerber, J. Garot, D. A. Bluemke, J. A. Lima, and O. A. Smiseth, "Quantitative assessment of intrinsic regional myocardial deformation by Doppler strain rate echocardiography in humans: validation against three-dimensional tagged magnetic resonance imaging," Circulation, vol. 106, pp. 50-6, 2002.

[7] P. Claessens, Claessens C. , Claessens J., Claessens M. , "Strain Imaging: Key to the Specific Left Ventricular Diastolic Properties in Endurance Trained Athletes," Journal of Clinical and Basic Cardiology, vol. 6, pp. 35-40 2003.

[8] M. Guarini, J. Urzua, A. Cipriano, and W. Gonzalez, "Estimation of cardiac function from computer analysis of the arterial pressure waveform," IEEE Transactions on Biomedical Engineering, vol. 45, pp. 1420-8, 1998.

[9] J. L. Palladino and A. Noordergraaf, "A paradigm for quantifying ventricular contraction," Cellular and Molecular Biology Letters, vol. 7, pp. 331-5, 2002.

[10] A. L. Hodgkin and A. F. Huxley, "A quantitative description of membrane current and its application to conduction and excitation in nerve," Journal of Physiology, vol. 117, pp. 500-44, 1952.

[11] G. W. Beeler and H. Reuter, "Reconstruction of the action potential of ventricular myocardial fibres," Journal of Physiology, vol. 268, pp. 177-210, 1977.

[12] C. H. Luo and Y. Rudy, "A dynamic model of the cardiac ventricular action potential. II. Afterdepolarizations, triggered activity, and potentiation," Circulation Research, vol. 74, pp. 1097113, 1994.

[13] R. L. Winslow, J. Rice, S. Jafri, E. Marban, and B. O'Rourke, "Mechanisms of altered excitationcontraction coupling in canine tachycardia-induced heart failure, II: model studies," Circulation Research, vol. 84, pp. 571-86, 1999.

[14] R. A. FitzHugh, "Impulses and physiological states in theoretical models of nerve membrane," Biophysical Journal, vol. 1, pp. 445-466, 1961.

[15] A. L. Bardou, P. M. Auger, P. J. Birkui, and J. L. Chasse, "Modeling of cardiac electrophysiological mechanisms: from action potential genesis to its propagation in myocardium," Critical Reviews ${ }^{\mathrm{TM}}$ in Biomedical Engineering, vol. 24, pp. 141-221, 1996.

[16] P. Colli Franzone, Pavarino L.F. and Taccardi B. , "Monodomain Simulations of Excitation and Recovery in Cardiac Blocks with Intramural Heterogeneity," Funcional Imaging and Modeling of the Heart (FIMH), pp. 267-277, 2005.

[17] C. S. Henriquez and R. Plonsey, "Simulation of propagation along a cylindrical bundle of cardiac tissue--I: Mathematical formulation," IEEE Transactions on Biomedical Engineering, vol. 37, pp. 850-60, 1990. 
[18] C. D. Werner, Sachse F.B. , and Dossel O., "Electrical excitation propagation in the human heart," International Journal of Bioelectromagnetism, vol. $2-2, \quad$ pp. http://www.rgi.tut.fi/ijbem/volume2/number2/werner/paper_ijbem.htm, 2000.

[19] P. C. Franzone, L. Guerri, M. Pennacchio, and B. Taccardi, "Spread of excitation in 3-D models of the anisotropic cardiac tissue. II. Effects of fiber architecture and ventricular geometry," Mathematical Biosciences, vol. 147, pp. 131-71, 1998.

[20] A. F. Huxley, "Muscle structure and theories of contraction," Progress biophysics and biophysical chemistry, vol. 7, pp. 255-318, 1957.

[21] J. J. Rice, R. L. Winslow, and W. C. Hunter, "Comparison of putative cooperative mechanisms in cardiac muscle: length dependence and dynamic responses," American Journal of Physiology, vol. 276, pp. H1734-54, 1999.

[22] J. Bestel, Clément F. , Sorine M., "A biomechanical model of muscle contraction," presented at Medical Image Computing and Computer-Assisted Intervention, 2001.

[23] J. J. Rice, M. S. Jafri, and R. L. Winslow, "Modeling short-term interval-force relations in cardiac muscle," American Journal of Physiology Heart and Circulatory Physiology, vol. 278, pp. H913-31, 2000.

[24] A. Y. Wong, "Myocardial mechanics: application of sliding-filament theory to isovolumic concentration of the left ventricle," Journal of Biomechanics, vol. 6, pp. 565-81, 1973.

[25] P. J. Hunter, A. D. McCulloch, and H. E. ter Keurs, "Modelling the mechanical properties of cardiac muscle," Progress in Biophysics \& Molecular Biology, vol. 69, pp. 289-331, 1998.

[26] P. H. Bovendeerd, P. Borsje, T. Arts, and F. N. van De Vosse, "Dependence of intramyocardial pressure and coronary flow on ventricular loading and contractility: a model study," Annals Biomedical Engineering, vol. 34, pp. 1833-45, 2006.

[27] R. C. Kerckhoffs, P. H. Bovendeerd, J. C. Kotte, F. W. Prinzen, K. Smits, and T. Arts, "Homogeneity of cardiac contraction despite physiological asynchrony of depolarization: a model study," annals of biomedical engineering, vol. 31, pp. 536-47, 2003.

[28] J. D. Humphrey, R. K. Strumpf, and F. C. Yin, "Determination of a constitutive relation for passive myocardium: II. Parameter estimation," Journal of Biomechanical Engineering, vol. 112, pp. 340-6, 1990.

[29] P. J. Hunter, "Myocardial constitutive laws for continuum mechanics models of the heart," Adv Exp Med Biol, vol. 382, pp. 303-18, 1995.

[30] V. P. Novak, F. C. Yin, and J. D. Humphrey, "Regional mechanical properties of passive myocardium," Journal of Biomechanics, vol. 27, pp. 403-12, 1994.

[31] I. Mirsky, "Assessment of passive elastic stiffness of cardiac muscle: mathematical concepts, physiologic and clinical considerations, directions of future research," Progress In Cardiovascular Diseases, vol. 18, pp. 277-308, 1976.

[32] R. C. Kerckhoffs, O. P. Faris, P. H. Bovendeerd, F. W. Prinzen, K. Smits, E. R. McVeigh, and T. Arts, "Timing of depolarization and contraction in the paced canine left ventricle: model and experiment," Journal of Cardiovascular Electrophysiology, vol. 14, pp. 188-95, 2003.

[33] K. May-Newman and A. D. McCulloch, "Homogenization modeling for the mechanics of perfused myocardium," Progress in Biophysics \& Molecular Biology, vol. 69, pp. 463-81, 1998.

[34] M. Sermesant, P. Moireau, O. Camara, J. Sainte-Marie, R. Andriantsimiavona, R. Cimrman, D. L. Hill, D. Chapelle, and R. Razavi, "Cardiac function estimation from MRI using a heart model and data assimilation: advances and difficulties," Medical Image Anals, vol. 10, pp. 642-56, 2006.

[35] F. J. Vetter and A. D. McCulloch, "Three-dimensional stress and strain in passive rabbit left ventricle: a model study," annals of biomedical engineering, vol. 28, pp. 781-92, 2000.

[36] M. B. B. L. G. Mohr, Seemann G., Sachse F.B. and Dössel O., "Volume Modeling of Myocard Deformation with a Spring Mass System," Lecture Notes in Computer Science, vol. 2673, pp. 332$339,2003$.

[37] B. Chahboune, Crolet J.M. , "Numerical simulation of the blood-wall interaction in the human left ventricle," European Physical Journal, vol. 2, pp. 291-297, 1998.

[38] P. R. Verdonck, Vierendeels J.A. , "Fluid-Structure Interaction Modelling of Left Ventricular Filling," International Conference on Computational Science, pp. 275 - 284, 2002.

[39] M. Sermesant, H. Delingette, and N. Ayache, "An electromechanical model of the heart for image analysis and simulation," ieee transactions on medical imaging, vol. 25, pp. 612-25, 2006. 
[40] R. C. Kerckhoffs, M. L. Neal, Q. Gu, J. B. Bassingthwaighte, J. H. Omens, and A. D. McCulloch, "Coupling of a 3D finite element model of cardiac ventricular mechanics to lumped systems models of the systemic and pulmonic circulation," annals of biomedical engineering, vol. 35, pp. 1-18, 2007.

[41] M. Courtois, S. J. Kovacs, Jr., and P. A. Ludbrook, "Transmitral pressure-flow velocity relation. Importance of regional pressure gradients in the left ventricle during diastole," Circulation, vol. 78, pp. 661-71, 1988.

[42] D. Nickerson, N. Smith, and P. Hunter, "New developments in a strongly coupled cardiac electromechanical model," Europace, vol. 7 Suppl 2, pp. 118-27, 2005.

[43] S. W. Ubbink, P. H. Bovendeerd, T. Delhaas, T. Arts, and F. N. van de Vosse, "Towards modelbased analysis of cardiac MR tagging data: relation between left ventricular shear strain and myofiber orientation," Medical Image Anals, vol. 10, pp. 632-41, 2006.

[44] T. Desaive, Ghuysen, A., Lambermont, B., Kolh, P., Dauby P.C., Starfinger, C., Hann, C.E., Chase, J.G. Shaw, G.M., "Study of ventricular interaction during pulmonary embolism using clinical identification in a minimum cardiovascular system model," presented at Conference of the IEEE Engineering in Medicine and Biology (EMB), Lyon France, 2007.

[45] C. E. Silva, L. D. Ferreira, L. B. Peixoto, C. G. Monaco, M. A. Gil, and J. Ortiz, "Study of the myocardial contraction and relaxation velocities through Doppler tissue imaging echocardiography: A new alternative in the assessment of the segmental ventricular function," Arquivos Brasileiros de Cardiologia, vol. 78, pp. 200-11, 2002.

[46] M. D. Cerqueira, N. J. Weissman, V. Dilsizian, A. K. Jacobs, S. Kaul, W. K. Laskey, D. J. Pennell, J. A. Rumberger, T. Ryan, and M. S. Verani, "Standardized myocardial segmentation and nomenclature for tomographic imaging of the heart. A statement for healthcare professionals from the Cardiac Imaging Committee of the Council on Clinical Cardiology of the American Heart Association," International Journal of Cardiovascular Imaging, vol. 18, pp. 539-42, 2002.

[47] V. Szathmary and R. Osvald, "An interactive computer model of propagated activation with analytically defined geometry of ventricles," Comput Biomed Res, vol. 27, pp. 27-38, 1994.

[48] L. A. Taber, M. Yang, and W. W. Podszus, "Mechanics of ventricular torsion," Journal of Biomechanics, vol. 29, pp. 745-52, 1996.

[49] A. I. Hernandez, "Fusion de signaux et de modèles pour la caractérisation d'arythmies cardiaques." thesis of Rennes university (France), 2000.

[50] A. I. Hernandez, G. Carrault, F. Mora, and A. Bardou, "Model-based interpretation of cardiac beats by evolutionary algorithms: signal and model interaction," Artificial Intelligence in Medicine, vol. 26, pp. 211-35, 2002.

[51] D. Durrer, R. T. van Dam, G. E. Freud, M. J. Janse, F. L. Meijler, and R. C. Arzbaecher, "Total excitation of the isolated human heart," Circulation, vol. 41, pp. 899-912, 1970.

[52] H. R. B. Chaudhry, B.; Findley T., "Stresses and Strains in the Passive Left Ventricle," Journal of Biological Systems, vol. 4, pp. 535-554, 1996.

[53] L. Back, "Left ventricular wall and fluid dynamics of cardiac contraction," Mathematical Biosciences, vol. 36, pp. 257 -297, 1977.

[54] M. Takata, Y. Harasawa, S. Beloucif, and J. L. Robotham, "Coupled vs. uncoupled pericardial constraint: effects on cardiac chamber interactions," Journal of Applied Physiology, vol. 83, pp. 1799-813, 1997.

[55] M. Ursino and E. Magosso, "Acute cardiovascular response to isocapnic hypoxia. I. A mathematical model," American Journal of Physiology Heart and Circulatory Physiology, vol. 279, pp. H149-65, 2000 .

[56] F. Wendling, Hernandez, A.I., Bellanger, J.-J., and P. Chauvel, and Bartolomei, F., "Interictal to Ictal Transition in Human Temporal Lobe Epilepsy: Insights From a Computational Model of Intracerebral EEG," Journal of Clinical Neurophysiology, vol. 22, pp. 343-356, 2005.

[57] D. D. Streeter, Jr. and W. T. Hanna, "Engineering mechanics for successive states in canine left ventricular myocardium. I. Cavity and wall geometry," Circulation Research, vol. 33, pp. 639-55, 1973.

[58] J. H. Holland, Adaptation in natural and artificial systems: MIT press, 1975.

[59] D. Beasley, Bull D.R., Martin R., "An overview of genetic algorithms : Part1, Fundamental," Univ. Comp., vol. 15, pp. 170-181, 1993. 
:19 [60] D. E. Goldberg, Genetic Algorithms in search, optimization and machine learning. Boston: Kluwer

[61] Z. Michalewicz, Genetic Algorithms + Data Structures = Evolution Programs. New-York: Springer-Verlag, 1994.

[62] F. L. Bookstein, "Principal Warps: Thin-Plate Splines and the Decomposition of Deformations," IEEE trans. Pattern Analysis and Machine Intelligence, vol. 11, pp. 567-585, 1989.

[63] C. M. Yu, J. Gorcsan, 3rd, G. B. Bleeker, Q. Zhang, M. J. Schalij, M. S. Suffoletto, J. W. Fung, D. Schwartzman, Y. S. Chan, M. Tanabe, and J. J. Bax, "Usefulness of tissue Doppler velocity and strain dyssynchrony for predicting left ventricular reverse remodeling response after cardiac resynchronization therapy," American Journal of Cardiology, vol. 100, pp. 1263-70, 2007.

[64] J. Beshai, R. A. Grimm, S. F. Nagueh, J. H. Baker, S. L. Beau, S. M. Greenberg, L. A. Pires, and P. J. Tchou, "Cardiac-Resynchronization Therapy in Heart Failure with Narrow QRS Complexes," New England Journal of medicine vol. 357, pp. 2461-2471, 2007.

[65] K. Dauterman, P. H. Pak, W. L. Maughan, A. Nussbacher, S. Arie, C. P. Liu, and D. A. Kass, "Contribution of external forces to left ventricular diastolic pressure. Implications for the clinical use of the Starling law," Annals of Internal Medicine, vol. 122, pp. 737-42, 1995. 
